\title{
Riflessioni sul linguaggio grafico nei poster di Savignac
}

\author{
Marcello Scalzo
}

\section{Abstract}

Nel primo dopoguerra in Francia, negli atelier dei grandi maestri quali Paul Colin e Cassandre, si forma una nuova generazione di affichiste, tra i quali Raymond Savignac e Bernard Villemot. Le novità nell'ambito della grafica che caratterizzeranno queste nuovi cartellonisti, sono la sintesi della comunicazione, il disegno essenziale e bidimensionale, il tratto deciso, i colori vivaci e una preferenza verso rapporti semplici che conducono all'immediata comunicazione del prodotto.

Nelle opere di Savignac, a partire dal secondo dopoguerra, emerge una nuova peculiarità: un umorismo semplice, ma non banale, a volte ironico, molto efficace che si coniuga con rappresentazioni caratterizzate da leggerezza e luminosità. I tratti con cui le figure sono disegnate diventano ancor più essenziali, quasi 'elementari'; i colori usati sono vivaci, stesi 'di getto', senza mediazione.

Raymond Savignac sa trasmettere messaggi pubblicitari diretti e immediati, di facile comprensione, commercialmente efficaci; ha ideato personaggi e immagini iconiche, contribuendo alla creazione di una 'identità visiva' tra il soggetto del poster e il prodotto pubblicizzato.

\section{Parole chiave}

affiches, grafica francese, Raymond Savignac, cartellonismo, pubblicità.

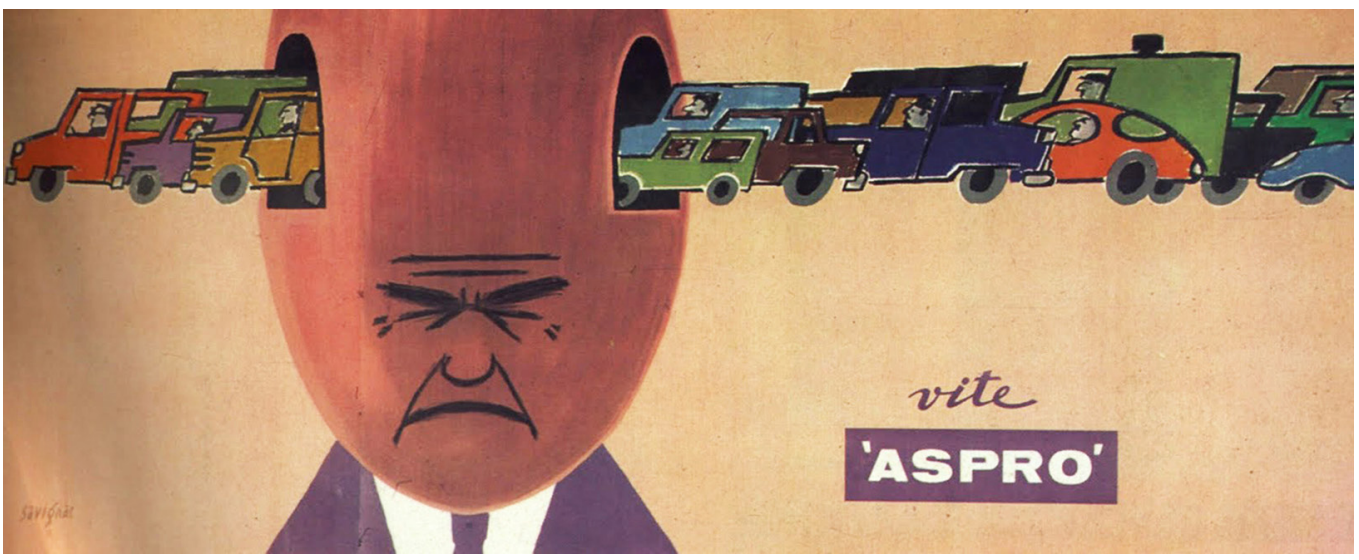


"A 12 anni dipingevo come Raffaello, poi ho impiegato una vita per imparare a dipingere come un bambino"

[Pablo Picasso]

\section{Premessa}

Savignac, è l'unico uomo conosciuto che può esibire due certificati di nascita. II primo, anagrafico, è datato al 6 novembre del 1907, del secondo ci racconta lui stesso: "Sono nato all'età di quarantun anni dalle mammelle della mucca Monsavon" [Savignac 1975, p. 9]. II 1949, data di pubblicazione del suo, forse, più famoso poster, è stato l'arrivo di un viaggio travagliato e non senza angosce, ma certo non privo di fascino. Savignac a differenza di altri artisti, non è stato un enfant prodige, non ha dimostrato un precoce talento per il disegno, né certezze sulla strada da intraprendere: la sua maturità artistica è arrivata dopo un lungo percorso di ricerca e di dubbi sul suo futuro professionale. Almeno sino alla sua 'seconda' nascita. Dal 1949 sino al 2002, anno della sua morte a quasi 95 anni, è stato un cartellonista di successo, prolifico, ricercato, produttivo e creativo sino all'ultimo.
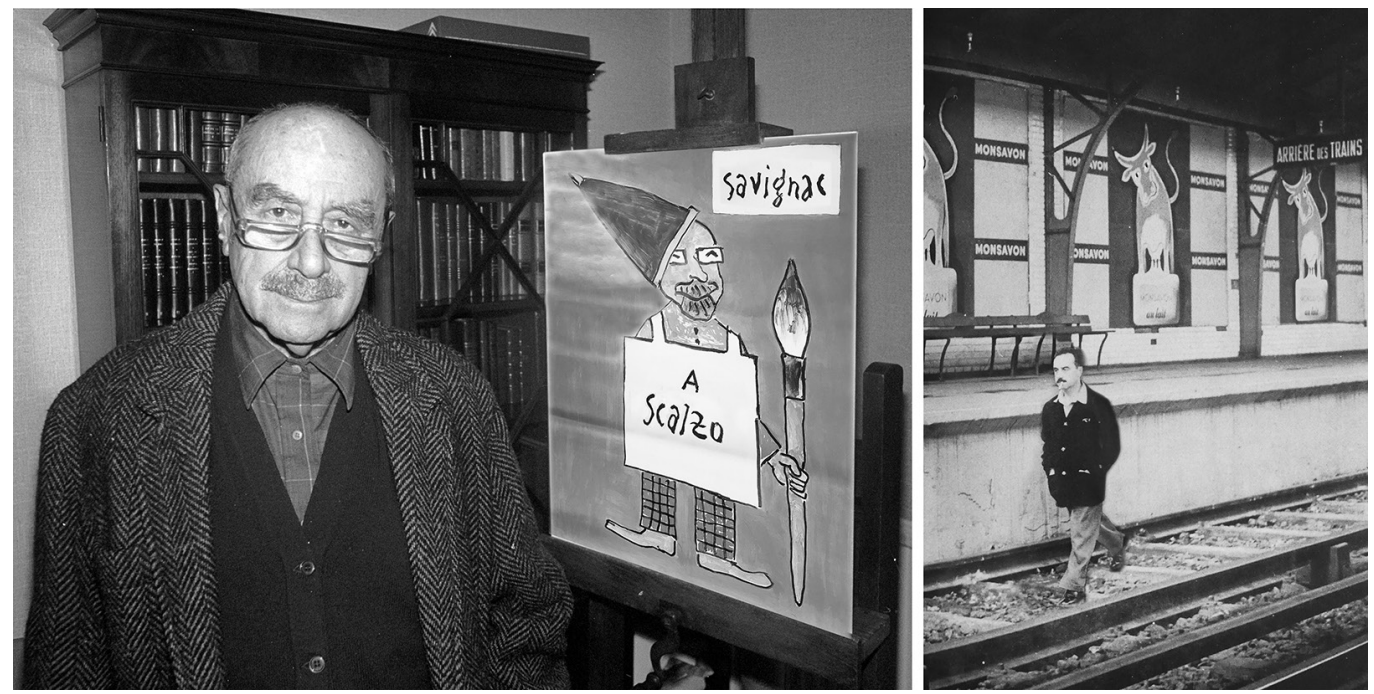

\section{Dalla prima alla seconda nascita}

Raymond Savignac nasce a Parigi il 6 novembre del 1907, al I 4 di rue Jeanne-Hachette, da genitori dell'Aveyron, un dipartimento francese della regione Occitania, trasferitisi nella capitale, dove aprirono un piccolo ristorante in rue de la Glacière. II giovane Raymond trascorre tutta la sua giovinezza nell'atmosfera dei caffè-ristoranti con la vocazione di diventare un ciclista, un'ambizione che ben presto si rivela del tutto irrealistica. II disegno lo interessa, ma come lui stesso scrive: "Pochi designer hanno avuto così poca vocazione come me" [Weill 1987, p. 5]. A differenza dei cartellonisti della sua generazione, Savignac non frequenta nessuna Accademia o Scuola d'arte [I], anzi è piuttosto negato per gli studi, infatti non porta neppure a termine un corso serale per disegnatore tecnico. Accantonato il sogno di diventare un ciclista professionista, nel 1923 all'età di 15 anni entra come copista nella Société des Transports en Commun de la Région Parisienne: il lavoro consiste nel ricalcare gli elaborati tecnici delle linee ferrotranviarie [Savignac 1975, p. 48]. La sera però nel bar dei suoi genitori si dedica con grande passione al disegno: realizza caricature, copia con perizia cartoline e fotografie. Alla S.T.C.R.P. Raymond entra in contatto con il più anziano disegnatore Marcel Foin che lo incoraggia nella sua carriera professionale: è lui che, qualche anno dopo, lo presenta al grafico Robert Lortac, che lo assume nel 1925 nel suo atelier di Montrouge [2]. Qui Savignac collabora alla realizzazione dei primi spot pubblicitari eseguiti con cartoni animati 

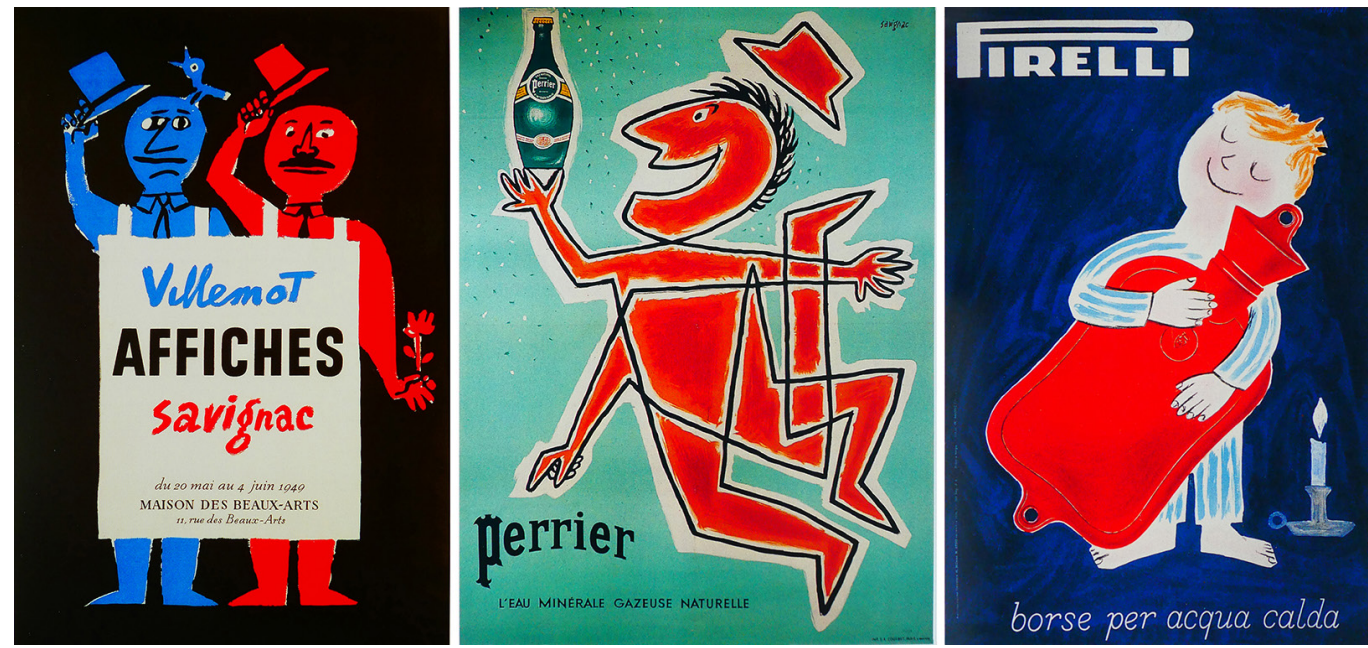

[Savignac 1975, p. 57]. La tecnica dei cartoon in Francia è ancora ai primordi, le figure per facilitarne la realizzazione, venivano mostrate quasi sempre di profilo, i personaggi erano molto semplici e articolati come marionette. Una grafica essenziale e minimalista, che ritornerà più avanti nello stile delle figure disegnate da Savignac. Nel 1927 parte per adempiere agli obblighi di leva e dopo aver terminato i 18 mesi di servizio militare, ritorna per un breve periodo nel laboratorio di Lortac; nel 1929 trova un impiego nella Ditta Miramar di Leven e Paulin, ma è un lavoro poco gratificante: disegna qualche etichetta, dipinge a mano scatole e coperchi. Sperimenta la tecnica della pittura con l'aerografo, ma se ne allontana ben presto, Savignac la ritiene una "macchina maledetta" che nelle sue mani non fa altro che "sputare e sbavare" [Savignac 1975, p. 86, Lelieur, Bacholet 200 I, p. 16]. Sono questi anni di crisi; infatti, dopo l'esperienza nella Miramar Savignac cambia spesso impiego, svolgendo lavoretti alternati alla disoccupazione, lavorando con lo stampatore Hacard, poi alla Métropole Publicité e realizzando inoltre alcuni poster per la Ditta Glasberg.

Nel tempo libero va al cinema, ne è affascinato, in particolare dalle gag comiche [Savignac 1975, pp. 62-63, Bauret 1982] di Charlie Chaplin, Buster Keaton, Harry Langdon, W.C. Fields e Harold Lloyd, ma, stranamente, non è interessato da quelle dei cartoni animati [3]. Nel 1933, in preda a dubbi e depressione, indeciso se proseguire la sua carriera di cartellonista, Savignac si reca nell'atelier di Cassandre [4], il direttore artistico della Alliance Graphique [5], per mostrare una cartella di suoi lavori. In quegli anni Cassandre è un grafico affermato, la sua Agenzia pubblicitaria ha ormai fama internazionale, produce poster per molti marchi in Europa e negli Stati Uniti. Lo scopo velato di Savignac è quello di chiedere all'affermato artista un parere sulle proprie capacità di cartellonista, se perseverare o abbandonare la professione, ed è pronto a sottomettersi al giudizio del più stimato collega [6].
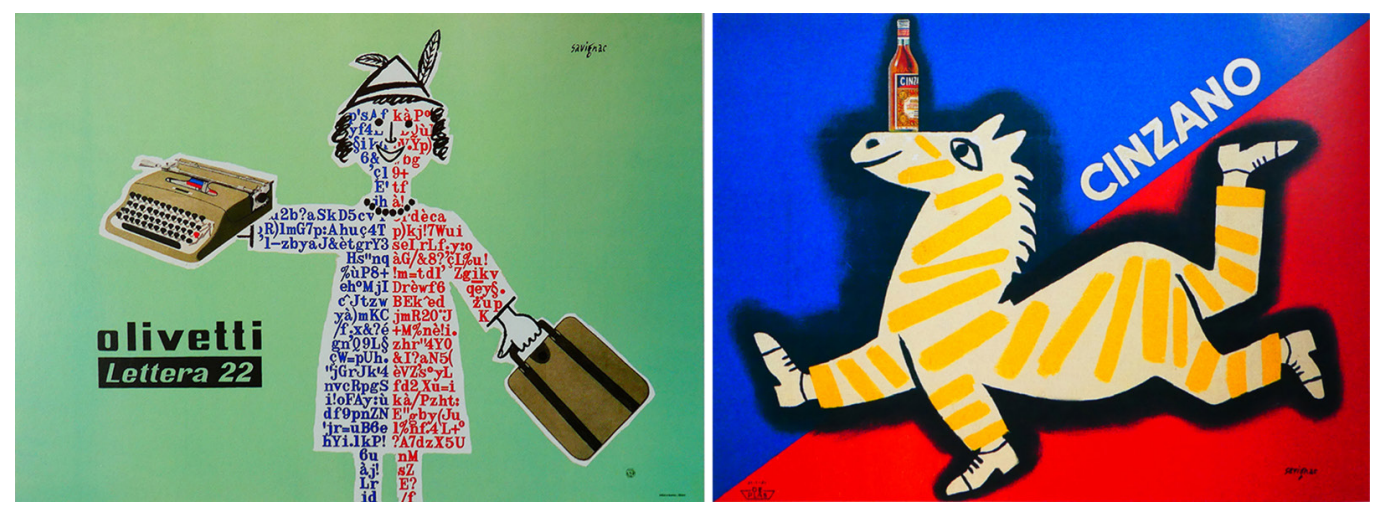
Fig. 4. Savignac: a sinistra, Margarina Gradina, 1953, al centro, Cafés Dya, 1959; a destra. Bel Paese Galbani, 1966.
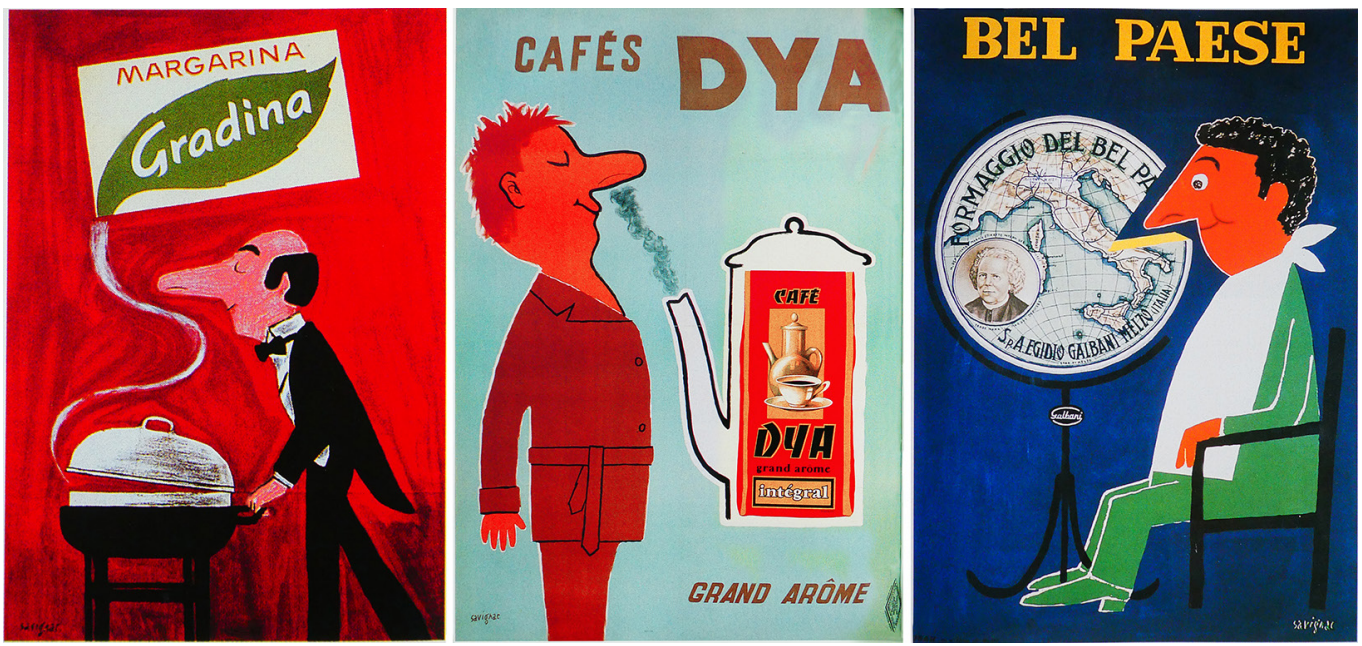

E invece esce dallo studio di rue Marc-Seguin, con due incarichi: eseguire un poster e un volantino [7]. Un buon inizio e man mano che il rapporto di collaborazione procede, acquista fiducia nelle proprie capacità, sino a divenire, dopo qualche tempo, assistente dell'affermato artista.

Cassandre per Savignac è un riferimento, un fratello maggiore, una guida, di lui scrive: "inconsapevolmente, è per me un esempio vivo e costante. Per imparare, devo solo guardarlo. Apro bene gli occhi e lo ascolto. Perché mentre lavora, parla. Parla ad alta voce, dice cosa farà, quali effetti intende ottenere, cosa ricerca [... . E questa disquisizione orale, che non è rivolta a me, è la migliore delle lezioni. Seguo i suoi ragionamenti e il suo singolare approccio ai problemi che deve risolvere" [Savignac 1975, p. 97]. Dal 1935 Cassandre propone a Savignac di collaborare direttamente con lui nel suo laboratorio a Versailles; benché non ne segnino la completa maturazione artistica, i cinque anni di collaborazione, lasciano un'impronta profonda nella formazione dell'artista.

Quando Cassandre nel 1938 parte per gli Stati Uniti, sponsorizza Savignac e ne favorisce l'impiego nella tipografia Draeger Frères.

Questo nuovo ambiente di lavoro, nonostante il buon stipendio, non piace a Raymond, trova l'atmosfera dello studio più simile a una caserma che a un laboratorio creativo, i suoi progetti non sono quasi mai considerati e, quando lo sono, vengono completamente stravolti [Weill 1987, p. 7, Lelieur, Bacholet 2001, p. 5 10].

Nel 1939 all'inizio del II conflitto mondiale Savignac viene richiamato e assegnato al Genio dell'8 ${ }^{\circ}$ Armata di stanza a Digione, prima come furiere, poi nel servizio di camouflage; mansioni di relativa tranquillità, tant'è che ricordando quel periodo Savignac arrivava a definirlo "gli anni della guerra divertente" [Savignac 1975, p. 125].
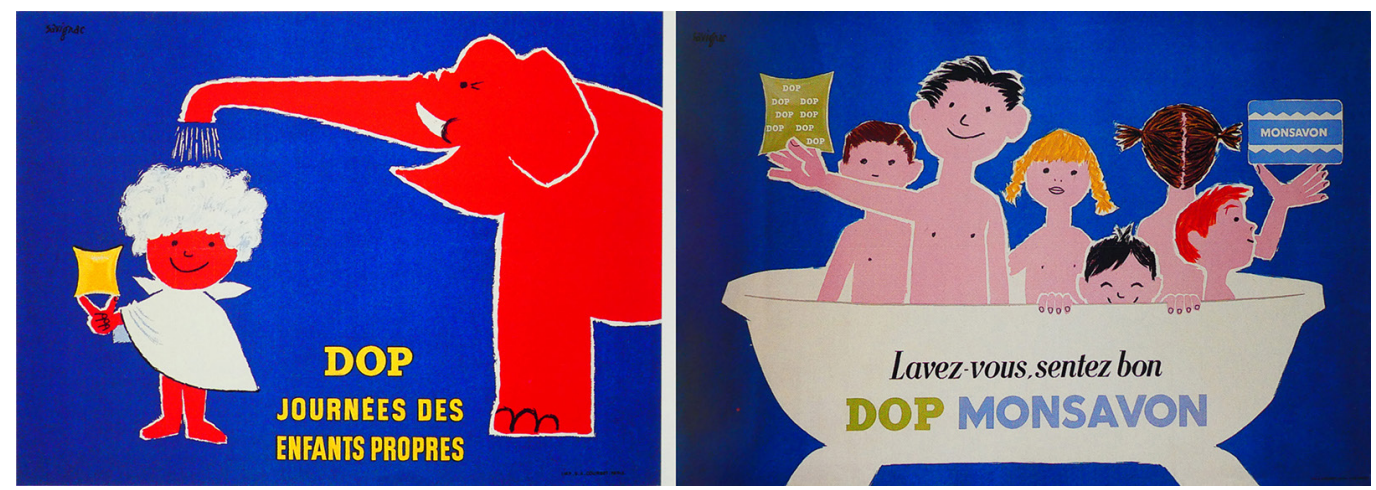


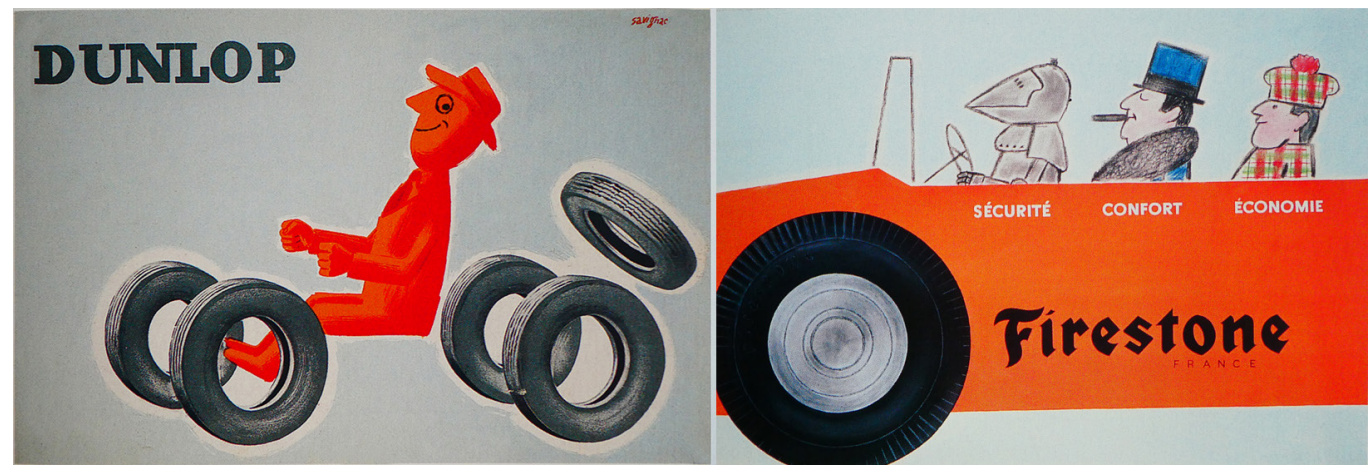

Congedato dopo la firma dell'armistizio Germania-Francia, nel giugno 1940 [8] ritorna a Parigi dove incontra il pubblicitario Robert Guérin, il factotum di Eugène Schueller, il proprietario de L'Oréal (e di Monsavon), che nel 1943 lo assume al Consortium Général de Publicité. Negli anni successivi realizza per il Consortium etichette, volantini, brochure, molti bozzetti, oltre ad alcuni poster: Armagnac Ryst, Armagnac Barnabé, birra Dalle; inoltre, illustra libri, disegna vignette per alcune riviste, realizza pagine pubblicitarie e copertine.

Nel 1947 il rapporto col Consortium si interrompe, l'anno successivo Savignac incontra Bernard Villemot [9]; questi, benché quasi suo coetaneo, è già un affermato cartellonista. Dalla Liberazione nel 1945, si è già fatto conoscere al grande pubblico, con alcuni allievi della scuola Paul Colin [I 0], di cui in gioventù è stato allievo, ha aperto uno studio di rue Danièle-Casanova. Bernard offre a Raymond la possibilità di trasferirsi nel suo atelier, qui Savignac si sente a proprio agio e vi resta per diversi anni.

Villemot agli inizi del 1949, in un momento di crisi di committenze, suggerisce al collega di preparare una mostra per promuovere alcuni dei loro lavori, bozzetti e manifesti. Savignac contatta un suo amico l'architetto Picot, conosciuto ai tempi della "guerra divertente", che gestiva una galleria annessa alla École des Beaux-Arts: ottiene la sala praticamente senza pagare nulla. Raymond disegna il poster per la mostra, utilizza solo tre colori: nero, blu e rosso.

Per far capire che espongono due autori, disegna due uomini vagamente somiglianti a loro uniti da un pannello a mo' di uomo sandwich. Nel pannello scrive i testi e sulla testa di uno dei due dipinge un uccellino; per caratterizzarli ulteriormente ne disegna uno coi baffi. Su questo particolare scrive Savignac: "NéVillemot né io abbiamo baffi. Quindi, per assomigliare al mio disegno, mi son lasciato crescere i miei. È proprio da allora che porto i baffi" [Savignac 1975, p. I 56]. La mostra viene inaugurata il 20 maggio 1949 e resta aperta sino 4 giugno.
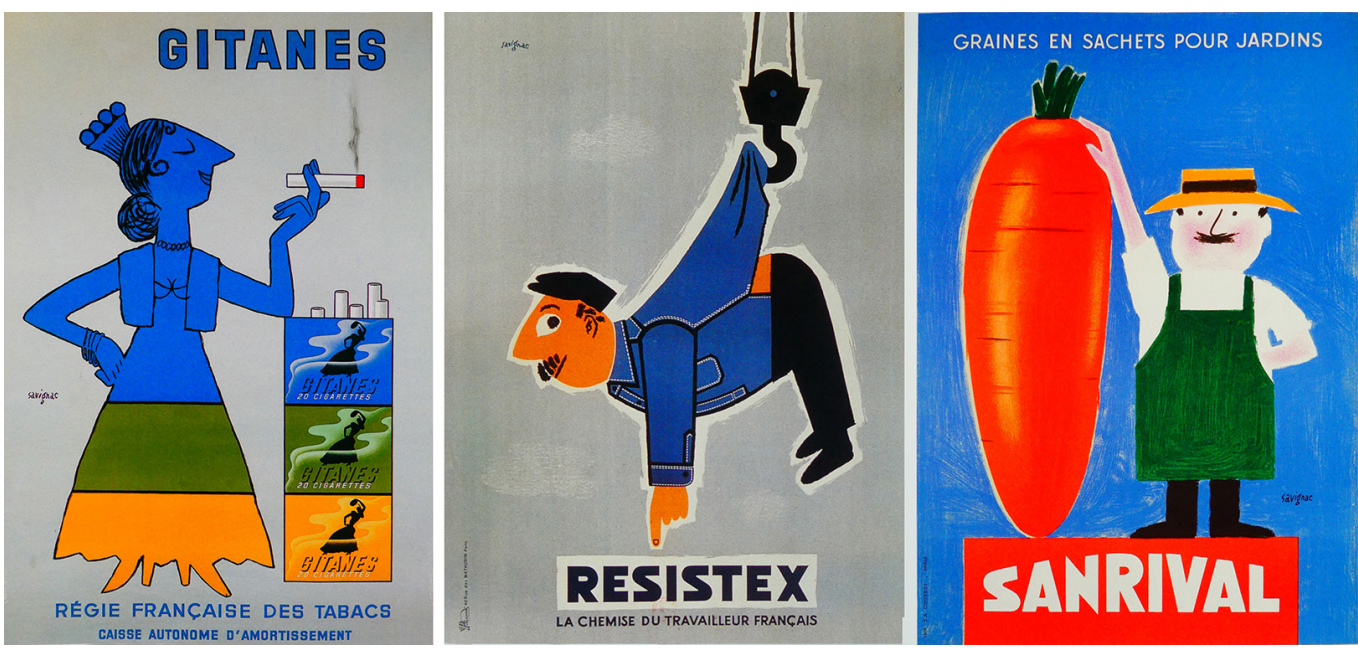
Per esporlo nella galleria, Raymond chiede a Robert Guérin un suo disegno per la pubblicità del Monsavon, bozzetto che in realtà giaceva già da diversi mesi in un cassetto del Consortium [l I]. Durante la mostra, il patron de L'Oreal, Eugène Schueller vedendolo esposto, ne resta folgorato e decide di darlo alle stampe immediatamente. II successo che ne segue è grandissimo e immediato. La città di Parigi viene invasa dalla mucca di Monsavon.

"Di solito inizio con due idee che si fondono in una sola. Quindi per Monsavon au lait, ho semplicemente pensato a un sapone per Monsavon e una mucca per il latte. Avrei quindi potuto sovrapporre un sapone su una mucca o viceversa: una banalità [...] Nel mio caso era il collegare questi due immagini così lontane con un elemento che dava vita e logica al mio poster. II latte che schizza dalle mammelle e si trasforma in sapone era il legame che lo rendeva evidente" [Lelieur, Bacholet 200 I, p. 24].

Savignac, dall'oggi al domani, diviene un cartellonista 'di grido' e inondato di ordini. In poco tempo il suo stile si afferma, viene osannato, imitato, entra nella storia dei poster, diventa da quel momento, con la sua forte personalità, un punto di riferimento di un'intera generazione di cartellonisti. Nella sua carriera Savignac ha lavorato per più di trecentocinquanta committenti [12] realizzando oltre 1000 tra manifesti, copertine e pagine pubblicitarie.
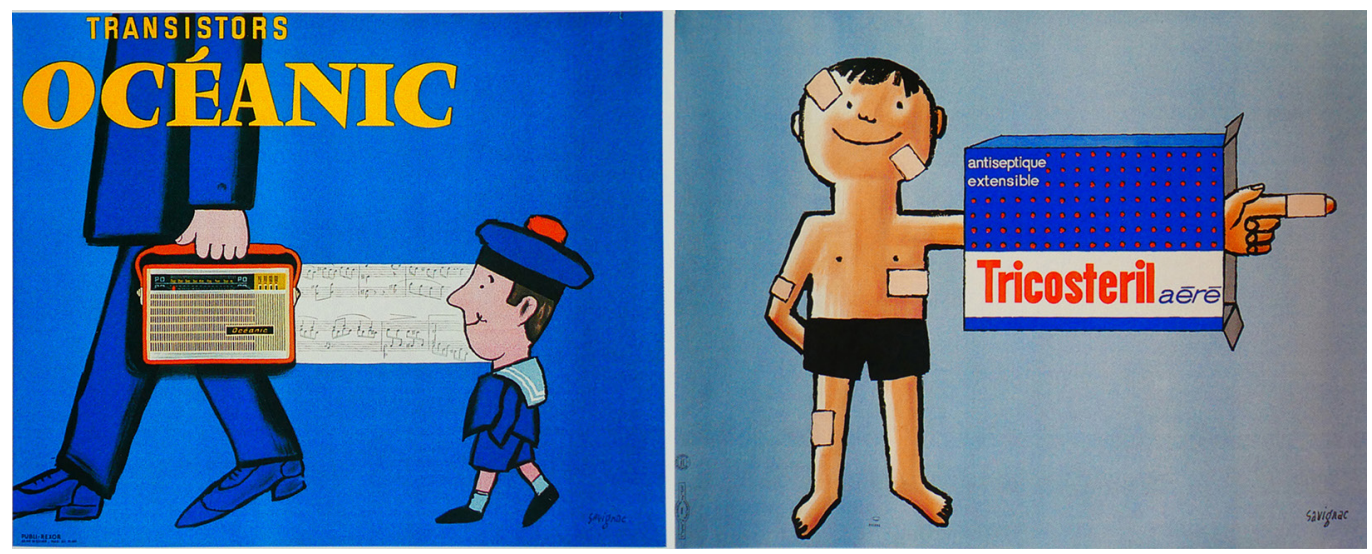

\section{II poster per Savignac}

Il poster è effimero. Come un giornale che viene buttato via dopo essere stato letto, il manifesto viene strappato, lacerato, coperto, sostituito, dimenticato dopo aver adempiuto alla sua funzione: ispirare un certo gesto o un'azione in chi lo ha guardato. La prima legge del poster, quindi, è balzare agli occhi, la seconda è che deve essere assimilato e compreso senza sforzo. Un'efficace pubblicità deve essere subito notata, può anche essere aggressiva, ma non deve né confondere, né respingere, né provocare scandalo; va invece assimilata e acquisita quasi nello stesso momento in cui viene osservata per la prima volta.

Nella nostra società, il poster non è usato solo per vendere, ma per far sognare le persone, ci fa desiderare o respingere qualcosa, altro non è che un modo per orientare il nostro desiderio. "Come l'opera d'arte, il poster è legato alla forza attrattiva dell'immagine. Ma, a differenza dell'opera d'arte, il poster deve essere visto e letto lo stesso giorno" [Gaëtan-Picon 1978, p. 7]. Savignac lavora da solo, "non crede nell'efficacia di un'idea pubblicitaria partorita da un gruppo, in una sorta di brainstorming all'americana" [Citéra 1998, p. 5], è fondamentalmente un anarchico [I3]. Non ha allievi, non gli interessa l'insegnamento, "prima di tutto perché non ho pazienza, e poi perché non credo affatto nella didattica in questo campo. Se un individuo è intelligente, sensibile, dotato, non ha alcun bisogno degli altri. Se è piuttosto limitato, la cosa non cambia" [Bauret 1982]. La forza di un poster si concretizza nell'impresa del singolo, che è allo stesso tempo designer, grafico, disegnatore, autore dello slogan e ideatore dell'immagine. 
La sua concezione della comunicazione è chiara: "ll poster non dovrebbe essere solo la trasposizione grafica di un'idea pubblicitaria, ma, prima di tutto, un messaggio di ottimismo; rivolgendosi al grande pubblico, non può preoccuparsi delle reazioni individuali, è quindi condannato a un linguaggio schematico, chiaro e brutale, che non lascia spazio ad aneddoti e sottigliezze" [Lelieur, Bacholet 200 I, p. 16].

Savignac può essere considerato l'inventore della 'gag grafica' o 'gag visiva', operazione che consiste nel pubblicizzare e vendere attraverso la gioia, al piacere della risata; lui stesso afferma, "quello che ho cercato di inserire nei miei poster è il buon umore" [Weill 1987, p. I I ]. Lo sviluppo di una "gag grafica" è un esercizio di grande rigore e alta acrobazia: il poster deve contenere un umorismo semplice, ma non banale e, a volte, ironico. Se un grande attore deve far esplodere lo schermo, il buon pubblicitario deve 'sfondare il muro' [Lescure 20 I 4, p. 54]. La 'gag visiva', in fondo, è un mezzo di scrittura grafica per provocare uno shock nei passanti, sia per la curiosità che può generare, sia per l'allegria che può provocare. La pubblicità deve comunque rimanere un'immagine, non essere troppo intellettuale ed essere allegra ed energica: "Dobbiamo tornare a cose semplici, quasi elementari, per ritrovare la salute" [Lelieur, Bacholet 200 I, p. 23].

Savignac non ha mai cercato di essere uno storico della comunicazione, un sociologo o un teorico, anche se il suo discorso rivela una riflessione organizzata sulla società e sul modo di convincere o condizionarla attraverso la pubblicità. Ė stato e resterà sempre e solo un cartellonista.
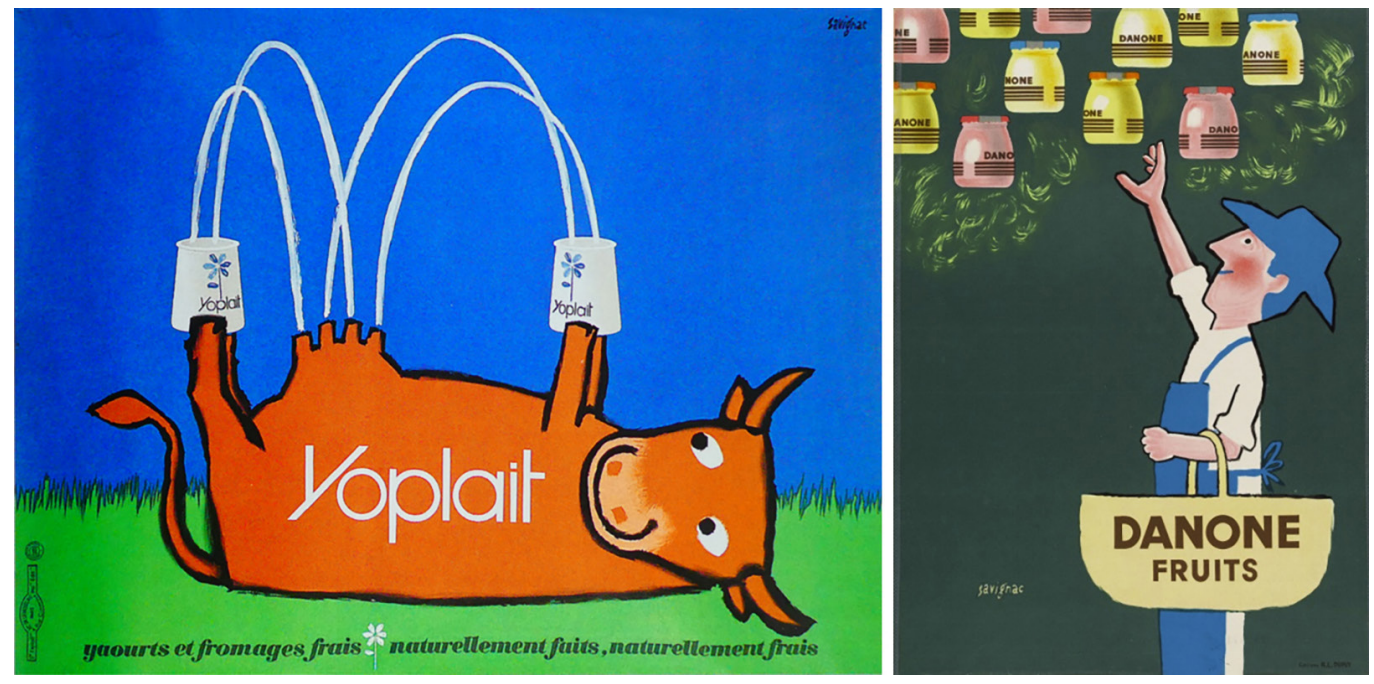

\section{Il disegno per Savignac}

Per Savignac il disegno è "la spina dorsale" del poster, deve essere dinamico, per dare vita all'idea. I suoi manifesti sembrano quasi degli abbozzi, schematici; le sue figure sono circondate da una linea nera, sembrano quasi ricordare i libri da colorare per bambini. Nelle sue opere il superfluo è assente, cancellato, sembra quasi che alcuni dei suoi poster siano già finiti con il solo disegno: "Meno mostriamo, più diciamo"' [Weill 1987, p. 40].

Savignac ha compreso che, per farsi capire, è opportuno dimenticare le sfumature, il realismo dei dettagli, in favore di un disegno essenziale, immediatamente leggibile, che mostri solo lo stretto necessario.

Ma Savignac è anche un talentuoso colorista, il colore è un elemento che si aggiunge al segno per renderlo più vigoroso e piacevole. Sa impostare i suoi personaggi su uno sfondo che li faccia risaltare: blu, rosso o giallo, colori base, ma lavorati sapientemente, cercando di 
Fig. 10. Savignac: a sinistra, Frigéco, 1958; a destra, Laden automatique, 1965.
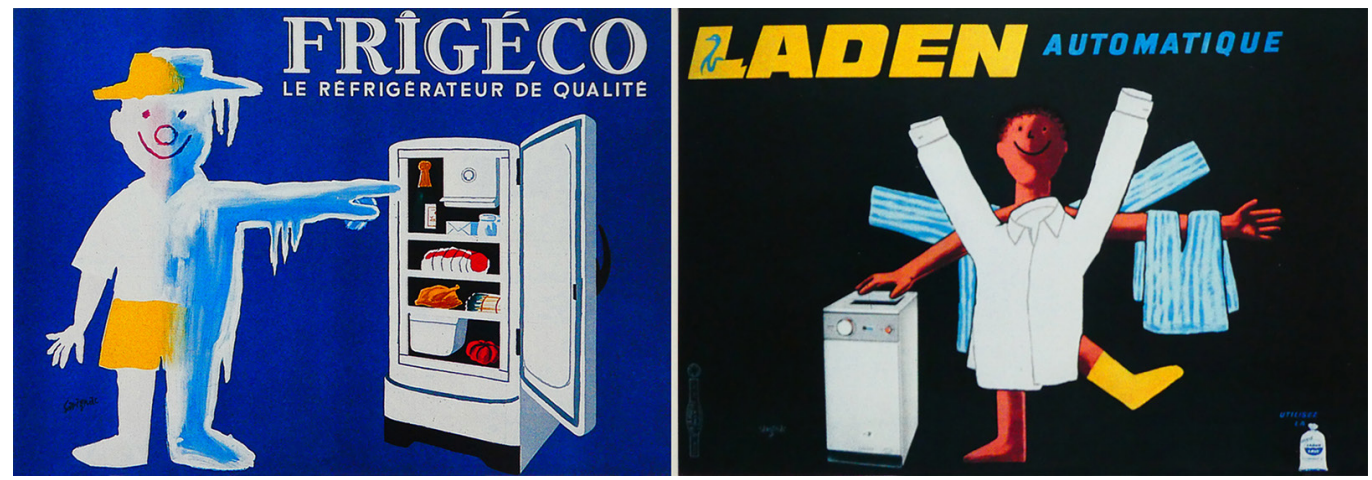

armonizzare le tinte con il prodotto da pubblicizzare. Gioca a 'colorare' i suoi disegni, come i bambini nei loro album [Weill 1987, p. 9].

In molte delle sue figure possiamo notare la quasi scomparsa degli effetti di rilievo, i personaggi si stagliano sullo sfondo evidenziati da un alone bianco o nero. I suoi punti deboli sono paradossalmente quelli di forza, il suo stile naif, ad esempio; lui stesso si definisce "grezzo e primitivo" Weill 1987, p. 8], in questo sta il primo segreto di Savignac. A questo scopo, i suoi layout sono assolutamente semplici, ormai lontanissimi dalle elaborate composizioni del suo maestro Cassandre. La schematicità dell'impostazione grafica dei poster di Savignac sembra richiamare quasi al concetto dell'ideogramma:"non l'ho inventato io, è antico quanto la Cina stessa. Sia Cassandre che Carlu già l'avevano sperimentato: quando cominciava a svilupparsi nella stampa il sistema del disegno senza didascalia" [Bauret 1982; Weill 1987, p. 9]. Savignac dà l'impressione che il manifesto sia disegnato da un bambino, il tratto della matita è quasi infantile, ma di grande autenticità; una freschezza, una spontaneità che tutti perdono quando diventano adulti. Lui l'ha riscoperta con tutta la scienza di un grafico esperto e il suo disegno non cambia nel tempo.

La buona pubblicità deve intrattenere l'utente con l'ausilio di immagini d'effetto che, a volte, possono essere associate a buoni slogan. Ma se il poster con la sola forma centra il risultato, il testo dello slogan diventa addirittura superfluo. L'essenza semplice, quasi infantile del linguaggio grafico di Savignac è di facile, diretta e immediata comprensione: e se un messaggio pubblicitario può essere capito da un bambino, arriverà sicuramente a tutti.

\section{Conclusioni}

Savignac introduce nello stile del moderno cartellonismo idee originali, nuovi schemi e modelli di comunicazione; veicola il messaggio commerciale con un linguaggio essenziale, al fine di una immediata leggibilità.
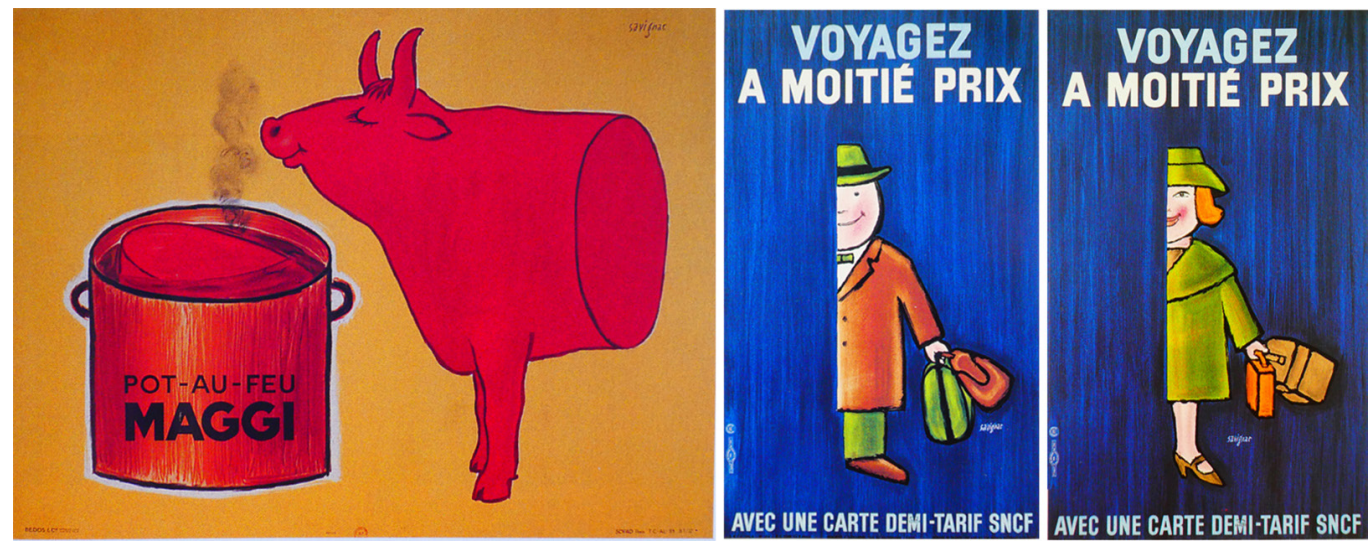

Fig. I I. Savignac: a sinistra Brodo Maggi, 1959; a centro, Viaggi a metà prezzo SNCF, 1964 prezzo SNCF, 1964 
Il disegno è alla base, la rappresentazione deve essere sintetica, senza slogan (che però è insito nell'immagine), i colori devono essere vivaci per attrarre l'osservatore.

Savignac comunica con le sue divertenti 'gag grafiche' messaggi di ottimismo; i suoi personaggi hanno sempre volti sorridenti, trasmettono nella loro schematicità, serenità e indirizzano efficacemente il messaggio commerciale.

Savignac ha sempre rigettato la spersonalizzazione della pubblicità che si serve della fotografia come espediente comunicativo.

Parafrasando i suoi maestri ideali del cinema comico, quali Charlot e Buster Keaton, Raymond Savignac "ha passato la vita disegnando barzellette" [Olsen 2008, p. I2].

"Noi cerchiamo in primo luogo di far ridere i bambini e senza volerlo facciamo ridere anche i grandi.

Che ci possiamo fare se i grandi restano sempre bambini" [Stan Laurel \& Oliver Hardy].

Fig. 12. Savignac: a sinistra OMO, 1963; al centro, il Giorno, 1960; a destra Vespa, 1955.
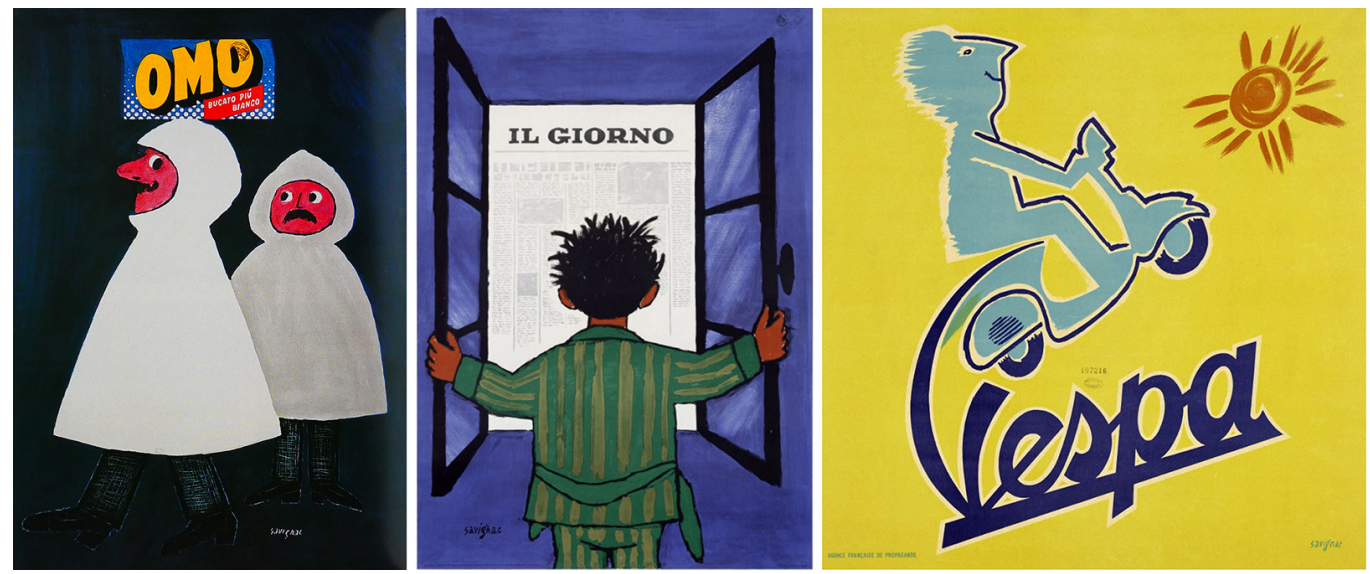

\section{Note}

[I] Dice Savignac "Sono un autodidatta, mi sono formato da solo disegnando di sera. Mi divertivo a riprodurre i disegni altrui: era bello e interessante" [Bauret 1982].

[2] Nell'atelier di Lortac Savignac inizia a comprendere l'opera dei cartellonisti: Cassandre, Loupot, Carlu, Cappiello e Derain, e impara a conoscere l'arte di Derain, Matisse, Picasso [Bauret 1982].

[3] L'unico cartone animato Savignac lo realizzerà nel 1959 per il Brodo Maggi [Lelieur, Bacholet 200 I, p. 58]

[4] Adolphe Jean-Marie Mouron, meglio noto come AM Cassandre, nasce nel I90 I a Charkiv, nell'Impero russo (ora Ucraina), da una famiglia di commercianti francesi; studia a Parigi pittura prima nell'atelier Lucien Simon, dopo nelle Accademie parigine de la Grande Chaumière e Julian. I suoi manifesti, tra gli anni 1920 e 1930, sono improntati sul movimento e la velocità, i suoi clienti sono: aziende ferroviarie, vagoni letto, navi di linea, automobili e biciclette. Per il grande pubblico, il suo nome rimane associato a una famosa campagna pubblicitaria del 1932, per un vino di china: Dubo, Dubon, Dubonnet in cui crea l'omino con la bombetta che si colora mentre beve il liquore. Nel 1938, ormai famoso con la sua agenzia l'Alliance Graphique, Cassandre si trasferisce negli USA per due anni. Tornato in Francia, oltre alla pittura e alla pubblicità, realizza costumi e scenografie teatrali. Suo è il famoso logo del 196 I per la maison Yves Saint Laurent. Depresso, in parte deluso, stressato dagli impegni lavorativi e dai clienti, muore suicida nel 1968

[5] L'Alliance Graphique era più un laboratorio creativo che un'agenzia pubblicitaria, era stata fondata nel 1930 da Cassandre, Charles Loupot e da Maurice Moyrand come direttore commerciale. Dopo l'improvvisa morte di quest'ultimo il I 5 settembre 1934, in un incidente stradale (Moyrand era il PR del gruppo, incaricato dei rapporti con i clienti e della vendita dei manifesti) e il progressivo defilarsi di Loupot, Cassandre sposta il suo laboratorio nella sua casa di Versailles.

[6] "Cassandre è l'unico il cui verdetto accetterò, qualunque esso sia" [Savignac 1975, p. 94].

[7] Cassandre deve aver percepito la determinazione del giovane Raymond, più giovane di sei anni, e subito gli affida alcuni incarichi: i suoi primi lavori nel 1933 sono il poster per il formaggio Roquefort Maria Grimal, Londres per Paul Morand e Cie du Chemin de Fer du Nord [Lelieur, Bacholet 2001, p. 92].

[8] Savignac il 22 febbraio del 1940 si era sposato con Marcelle Andréa Mercier, una modista conosciuta l'anno prima; di le dice "I'unica donna che crede nel mio talento" [Savignac 1975, p. I35]

[9] Bernard Villemot, o più semplicemente Villemot, nasce a Trouville-sur-mer nel 191।, il padre Jean era un illustratore e umorista: da bambino dimostra un vero talento per il disegno e dopo l'istruzione nelle scuole locali a Parigi e intraprende oli studi artistici presso l'Accademie Julian. Dopo il servizio di leva nel 1931, dal I932 al 1934 frequenta lo studio di Jean Colin. Sul 
finire del 1934 apre un suo atelier con alcuni allievi conosciuti nell'atelier di Colin. Dal 1935 inizia a realizzare i primi manifesti cinematografici e poster di esposizioni; negli anni ‘ 40 realizza alcuni manifesti per il Segretariato alla Gioventù e per il governo di Vichy. Ma è a partire dal 1945, con i poster La Pace ritrovata, che emerge il suo stile originale:Villemot firma le campagne pubblicitarie per la Croce Rossa e per temi sociali (aiuti ai bambini, lotte contro l'alcolismo, il cancro, l'assistenza ai reduci e ag anziani), per la Sanità pubblica, per le Forze Armate, per il Turismo. Le pubblicità commerciali lo consacrano al grande pubblico: Villemot realizza innumerevoli poster, rimasti nella memoria collettiva, per grandi marchi di tabacchi, elettrodomestici, alimentari, liquori, cosmetici, automobili, motori e tanto altro. Nel 1953, inizia la collaborazione, durata quasi vent'anni, con Orangina: la scorza d'arancia sbucciata tagliata a spirale resta per anni l'incontrastata icona della bibita. La campagna per la Perrier inizia nel 1956, segnata dal celebre slogan "c'est fou...". A partire dal 1969 lavora per Bally producendo alcuni storici manifesti: le famose losanghe arancioni, le lunghe gambe bianche, i fondi neri che diventano vestiti, le sinuose silhouette, restano alcuni tra i più belli della sua carriera. L'artista muore nel 1989 nella sua Trouville-sur-mer.

[ I 0] Paul Colin era un affermato cartellonista: nato a Nancy nel 1892 studia all'École des Beaux-Arts, è poi allievo di Eugène Vallin e di Victor Prouvé. Nel 1929 apre un proprio atelier; qui si formano artisti quali Villemot, Leupin, Henrion, Carrier, Derome e il duo Lefor-Openo. Nella sua carriera realizza cartelloni per teatro e cinema, locandine per spettacoli musicali e concerti, tra cui il famoso poster per la Revue nègre al Music-Hall con Joséphine Baker, di cui fu amante. Appassionato di musica e soprattutto di jazz, prolifico disegnatore, rappresenta i suoi personaggi, artisti, ballerini e cantanti, in una mirabile sintesi formale estremamente caratterizzante del personaggio. Cura importanti campagne pubblicitarie per la S.N.C.F., la Transatlantique, la Philips, la Columbia. Nel 1946 realizza il poster per il primo Festival di Cannes. Artista fecondo produsse circa 1900 tra poster, locandine, copertine di riviste. molte scenografie teatrali e costumi. Muore a Nogent-sur-Marne nel 1985.

[I I] Robert Guérin aveva commissionato qualche mese prima a Savignac il bozzetto per Monsavon au lait, il poster benché pagato, non era stato pubblicato, aveva destato qualche perplessità. Restò quindi nei cassetti de L'Oréal. Eugène Schueller nel visitare la mostra, arrivato davanti al poster della mucca, si girò verso Savignac e quasi commosso esclamò "Ah, Savignac, è meraviglioso il poster chi mi hai fatto" [Savignac 1975, p. I57].

[ I 2] Tra questi alcuni sono marchi di rilievo internazionale:Air France, Air Wick, André, Aspro, Astral, ATD, Bic, Cinzano, Citroën Collie Mischungs-Treu, Compagnie Wagons Lit France, Crédit Industriel et Commercial, Dalle, Danone, Dop, Dunlop, Dunlopillo, Eau écarlate, French Tobacco Company, Frigeco, Garap, II Giorno, Gendarmerie National, Laine d 'Aoust, Life, Lotterie National, Maggi, Michelin, Monsavon, Mobil, Océanic, Omo, Perrier, Petit Bateau, Pirelli, Point P., Renault, Résistex, Reynolds, Salveplast, Savings Bank, Seb, Solutricine, SNCF, Tréca, Triplex, Tricostéril, Ultracolor, Vérigoud, Vichy Célestins, Yoplait. Savignac lavora inoltre per Enti pubblici e Associazioni, per Movimenti d'opinione, come, ad esempio, il famoso poster realizzato per la campagna di sensibilizzazione "No alla superstrada sulla riva sinistra" del 197I.

[13] Così lo definisce A. Weill [Weill 1987, p. I3]. E in effetti nella professione Savignac ha un carattere decisamente anarchico: a parte il periodo in cui utilizza lo studio diVillemot, dal 1948 al |953, lavorerà sempre da solo. In apparenza non si interessa né politica [Savignac 1975, p. 5I], nè di satira politica "Il disegno politico non è il mio pezzo forte" [Savignac I975, p. I65]. Dodici

\section{Riferimenti bibliografici}

Bauret G. (1982). Intervista a Savignac. In Zoom, n. 22, settembre, p. 43 ss.

Choko M. H. (2005). Raymond Savignac. Paris: Pyramyd.

Citéra F. (1998). Savignac en vrac. Cabourg: Ed. Cahiers du Temps.

Decaen Le Boulanger H. (2008). La revolution Savignac. Cabourg: Ed. Cahiers du Temps.

Gaëtan-Picon G. (1978). In Musée de l'affiche. Paris: s.d. ma.

Lelieur A. C., Bacholet R. (200 I). Savignac affichiste. Paris: Ed. Bibliothèque Forney.

Lescure P., Buchard S. (20|4). Marques et Pubs cultes. Paris: Éd. De La Martinière.

Olsen R. (2008). Savignac s'affiche. Paris: Le Cherche midi.

Savignac R. (1975). Savignac affichiste. Paris: Ed. Robert Lafont.

Weil A. (1987). Savignac. L'affıche de A à Z. Montpellier: Seuil - Édition Hoëbeke.

\section{Autore}

Marcello Scalzo, Università degli Studi di Firenze, marcello.scalzo@unifi.it

Per citare questo capitolo: Scalzo Marcello (202I). Riflessioni sul linguaggio grafico nei poster di Savignac/Reflections on the graphic language of Savignac's poster. In Arena A., Arena M., Mediati D., Raffa P. (a cura di). Connettere. Un disegno per annodare e tessere. Linguaggi Distanze Tecnologie. Atti del $42^{\circ}$ Convegno Internazionale dei Docenti delle Discipline della Rappresentazione/Connecting. Drawing for weaving relationship. Languages Distances Technologies. Proceedings of the $42^{\text {th }}$ International Conference of Representation Disciplines Teachers. Milano: FrancoAngeli, pp. I I 23 - I I 42. 


\title{
Reflections on the Graphic Language of Savignac's Poster
}

\author{
Marcello Scalzo
}

Abstract

In the first post-war period in France, at Paul Colin and Cassandre atelier, a new generation of affichiste will take shape, among which Raymond Savignac and Bernard Villemot. The novelties in the field of graphics that characterize these poster designers are a very synthetic, two-dimensional, geometric graphic style, with strong chromatic contrasts and a preference for simple relationships that lead to the immediate communication of the product.

In the Savignac's works from the second post-war period a new peculiarity emerges: a simple, but not banal, sometimes ironic, very effective humor combined with representations characterized by lightness and brightness. The signs with which the figures are drawn become even more essential, almost 'elementary'. The colors used are bright, laid out 'quickly', without mediation.

Raymond Savignac is able to transmit direct and immediate advertising messages, easy to understand, commercially effective. He conceptualized iconic characters and images, contributing to the creation of a "visual identity" between the subject of the poster and the advertised product.

\section{Keywords}

affiche, french graphics, Raymond Savignac, poster, advertisement.

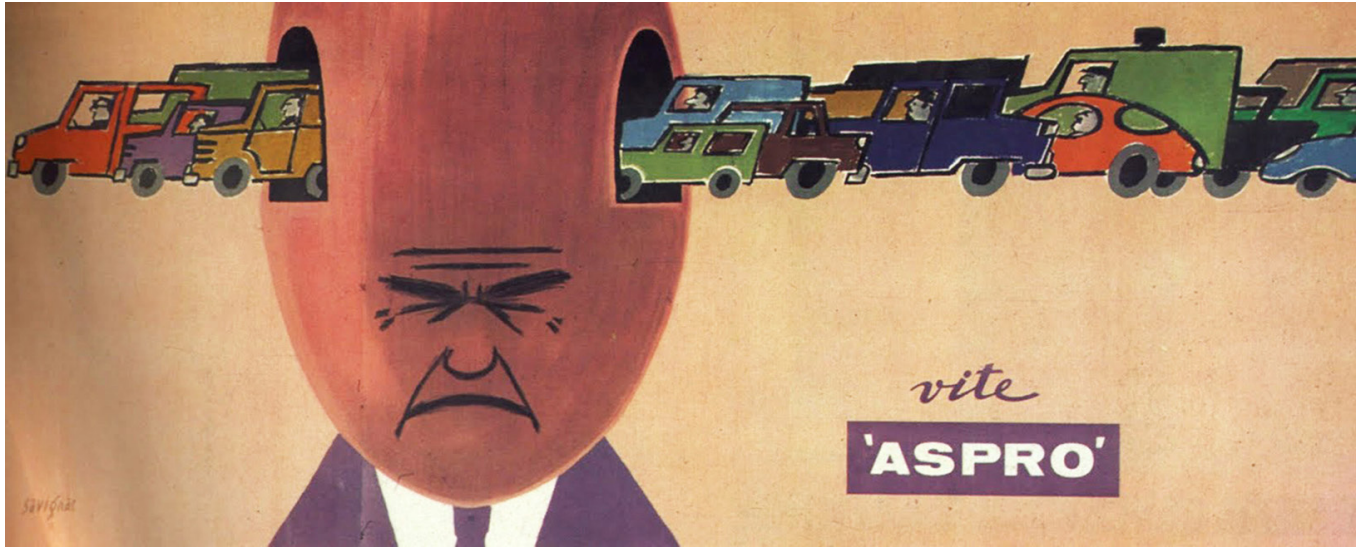


"A 12 anni dipingevo come Raffaello, poi ho impiegato una vita per imparare a dipingere come un bambino"

[Pablo Picasso]

\section{Premise}

Savignac is the only known man who can produce two birth certificates. The first is dated $6^{\text {th }}$ of November 1907, the second as he tells us: "Sono nato all'età di quarantun anni dalle mammelle della mucca Monsavon" [Savignac 1975, p. 9]. 1949, the year of publication of perhaps his most famous poster, was the arrival of a troubled journey, not without anguish, but certainly not without fascination. Unlike other artists, Savignac was not an enfant prodige, he did not show an early talent for drawing, nor certainty about the road to take: his artistic maturity came after a long journey of research and doubts about his professional future. At least until his "second" birth. From 1949 until 2002, the year of his death at almost 95 years of age, he was a successful, prolific, sought-after, productive and creative poster artist right up to the end.

Fig. I. Left, Raymond Savignac in the early 1990s; right, photomontage, Savignac in the early 1950s, behind him posters of Monsavon au lait.
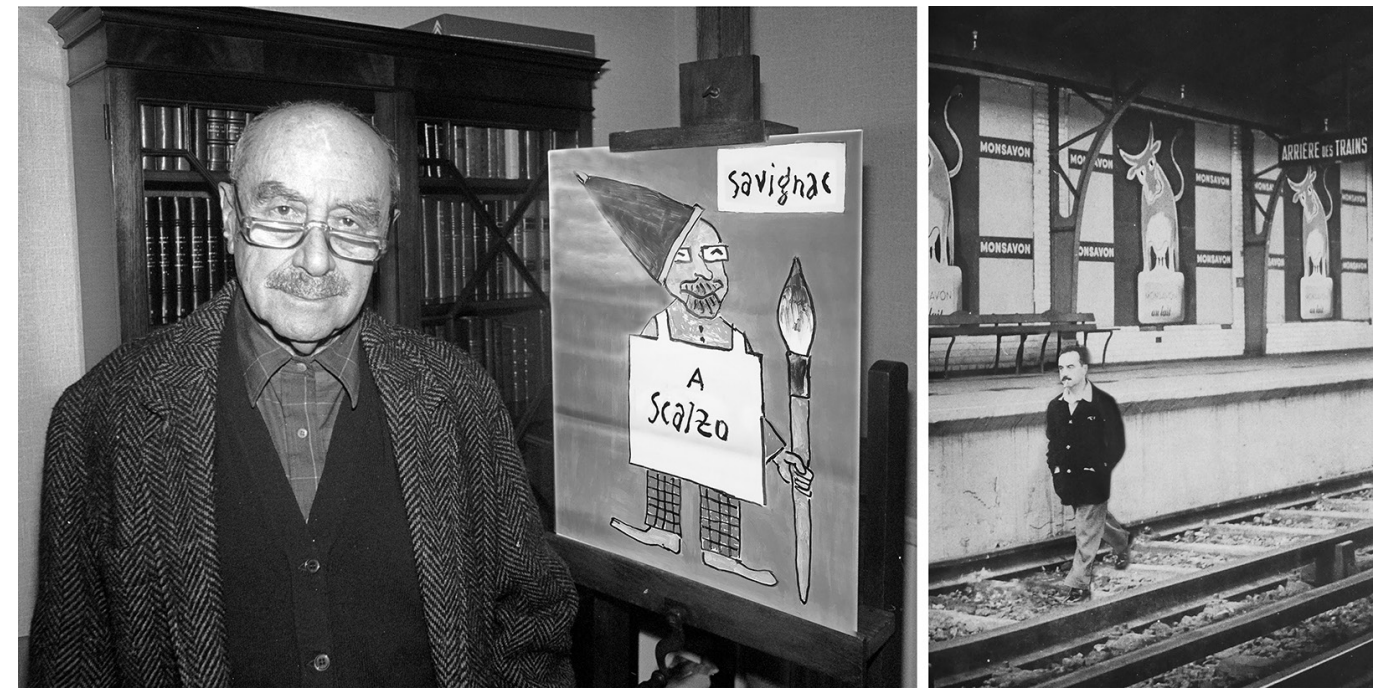

\section{From the first to the second birth}

Raymond Savignac was born in Paris on the $6^{\text {th }}$ of November 1907, at 14 rue JeanneHachette, to parents from Aveyron, a French department in the Occitane region, who moved to the capital, where they opened a small restaurant in rue de la Glacière. Young Raymond spent all his youth in the atmosphere of the café-restaurants with the vocation of becoming a cyclist, an ambition that soon turned out to be completely unrealistic. Drawing interested him, but as he wrote: "Pochi designer hanno avuto cosi poca vocazione come me" [Weill 1987, p. 5]. Unlike the poster designers of his generation, Savignac did not attend any academy or art school [I], and in fact he was rather bad at studies, not even completing an evening course for technical drawing. Having abandoned his dream of becoming a professional cyclist, in 1923, at the age of 15, he joined the Société des Transports en Commun de la Région Parisienne as a copyist: the job consisted of tracing the technical drawings of the railway lines [Savignac 1975, p. 48]. In the evenings, however, in his parents' bar, he devoted himself with great passion to drawing. He made caricatures and expertly copied postcards and photographs. At S.T.C.R.P. Raymond met the older draughtsman Marcel Foin, who encouraged him in his professional career: it was he who, a few years later, introduced him to the graphic designer Robert Lortac, who employed him in 1925 in his studio in Montrouge [2]. There Savignac worked on the first cartoon commercials [Savignac 1975, p. 57]. 

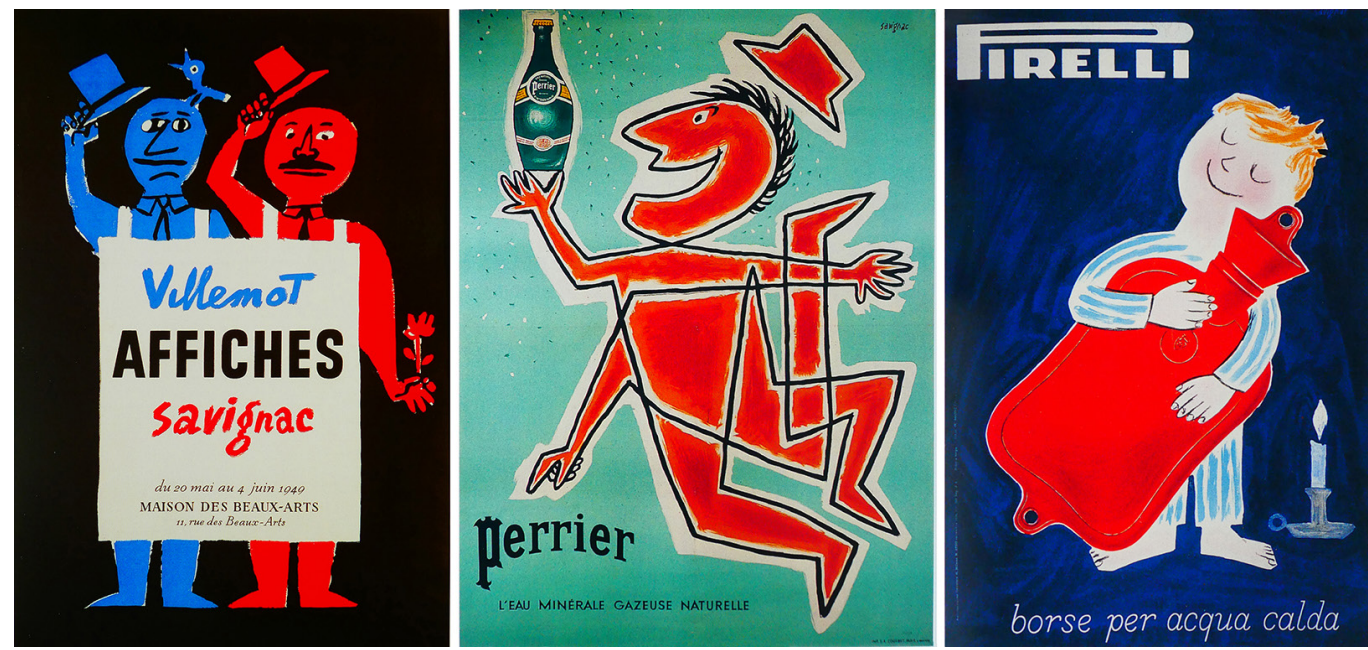

The technique of cartoons in France was still in its infancy, the figures were almost always shown in profile to facilitate their production, the characters were very simple and articulated like puppets. An essential and minimalist graphic style, which would return later in the style of the figures drawn by Savignac.

In 1927, he left to do his military service and after 18 months of military service, he returned to the Lortac workshop for a short period. In 1929, he found a job at the Miramar company of Leven and Paulin, but it was not very rewarding: he drew a few labels and hand-painted boxes and lids. He experimented with the technique of painting with an airbrush, but soon abandoned it, as Savignac considered it a "macchina maledetta" that in his hands did nothing but "sputare e sbavare" [Savignac 1975, p. 86; Lelieur, Bacholet 200 I, p. 16]. These were the years of crisis, and after his time at Miramar, Savignac often changed jobs, working alternately on and off the job market, working with the printer Hacard, then at Métropole Publicité, and producing posters for the Glasberg company.

In his free time, he went to the cinema and was fascinated by it, in particular by the comic gags [Savignac 1975, pp. 62-63; Bauret 1982] of Charlie Chaplin, Buster Keaton, Harry Langdon, W. C. Fields and Harold Lloyd, but strangely, he was not interested in cartoons [3]. In 1933, doubtful and depressed, unsure whether to continue his career as a poster artist, Savignac went to the atelier of Cassandre [4], the artistic director of the Alliance Graphique [5], to show a portfolio of his work. At that time Cassandre was an established graphic designer, his advertising agency was now internationally renowned, producing posters for many brands in Europe and the United States. Savignac's veiled aim was to ask the established artist for an opinion on his skills as a poster artist, whether to persevere or abandon the profession, and he was ready to submit to the judgement of his most esteemed colleague [6].
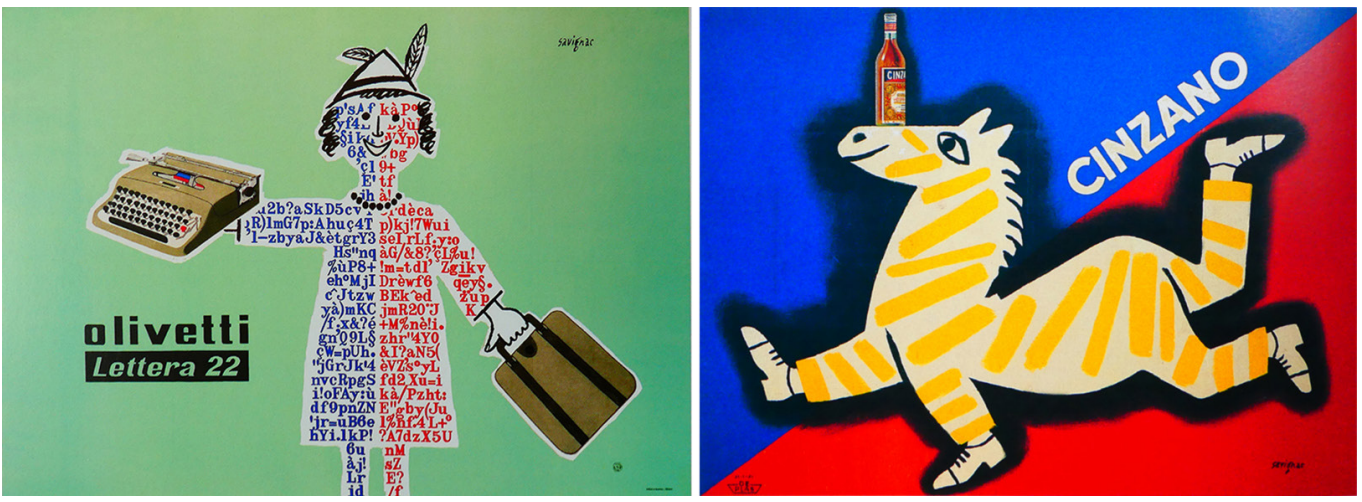
Fig. 4. Savignac: on the left, Margarina Gradina, 1953; in the centre, Cafés Dya, 1959. on the right Bel Pase
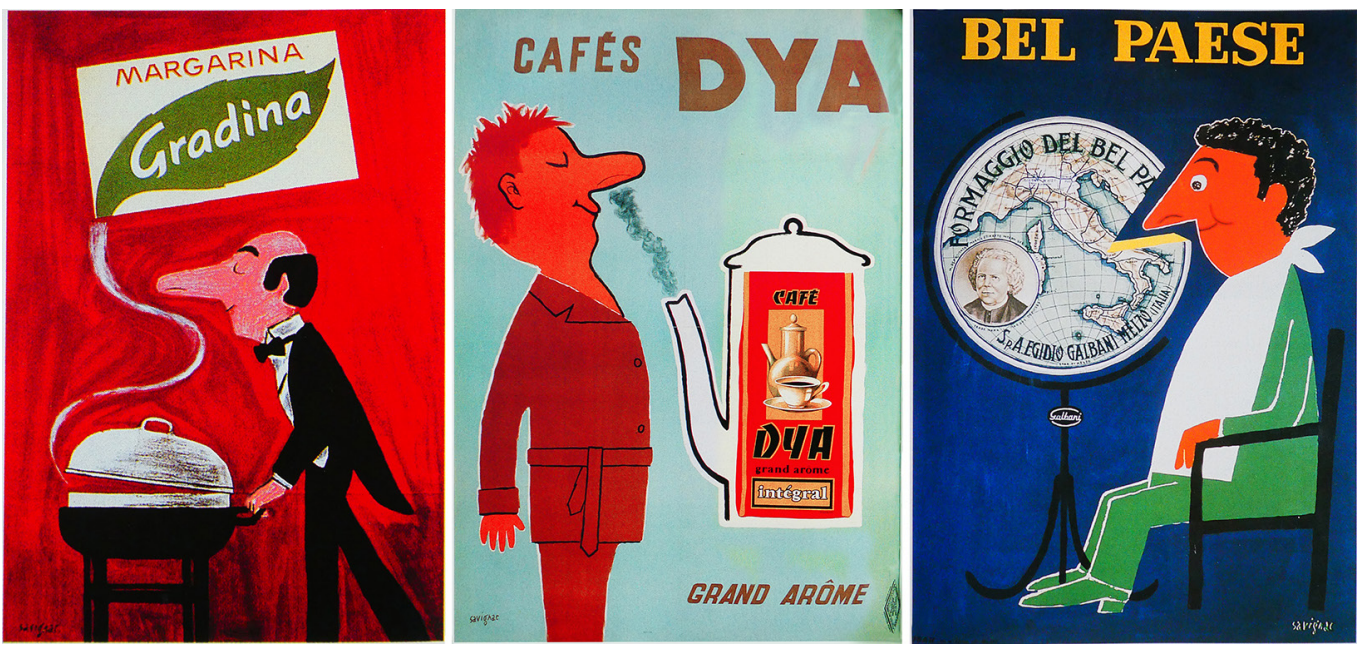

Instead, he left his studio in rue Marc-Seguin with two jobs: to produce a poster and a flyer [7]. It was a good start and as the collaboration progressed, he gained confidence in his own abilities and after a while became the assistant of the successful artist.

For Savignac, Cassandre is a reference, an older brother, a guide, and he writes of him: "inconsapevolmente, è per me un esempio vivo e costante. Per imparare, devo solo guardarlo. Apro bene gli occhi e lo ascolto. Perché mentre lavora, parla. Parla ad alta voce, dice cosa farà, quali effetti intende ottenere, cosa ricerca [...] E questa disquisizione orale, che non è rivolta a me, è la migliore delle lezioni. Seguo i suoi ragionamenti e il suo singolare approccio ai problemi che deve risolvere" [Savignac 1975, p. 97]. In 1935, Cassandre asked Savignac to work with him in his workshop in Versailles; although this did not mark his complete artistic maturity, the five years of collaboration left a deep impression on the artist's training.

When Cassandre left for the United States in 1938, he sponsored Savignac and favored his employment at the Draeger Frères typography.

This new working environment, despite the good salary, did not appeal to Raymond, who found the atmosphere of the studio more like a barracks than a creative workshop, his projects were hardly ever considered and, when they were, they were completely overturned [Weill 1987, p. 7, Lelieur, Bacholet 200 I, p. 5 I0].

In 1939, at the beginning of the Second World War, Savignac was called up and assigned to the 8th Army Corps of Engineers stationed in Dijon, first as a guardsman, then in the camouflage service; relatively quiet tasks, so much so that Savignac would go so far as to describe this period as "gli anni della guerra divertente" [Savignac 1975, p. 125].

Discharged after the signing of the Germany-France armistice, in June 1940 [8] he returned to Paris where he met the advertising executive Robert Guérin, the factotum of Eugène
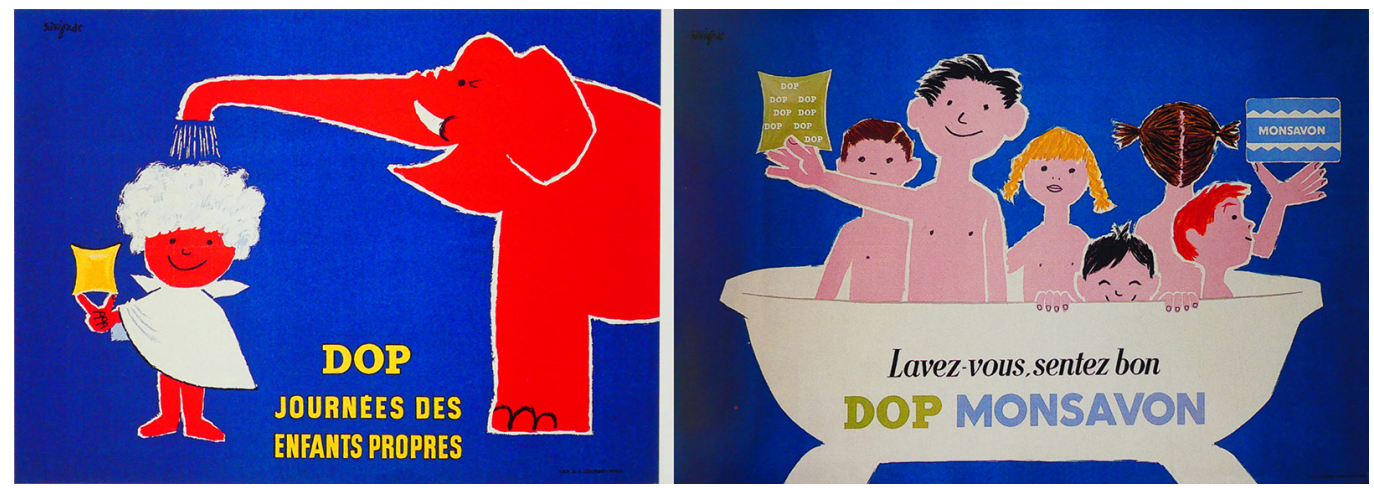


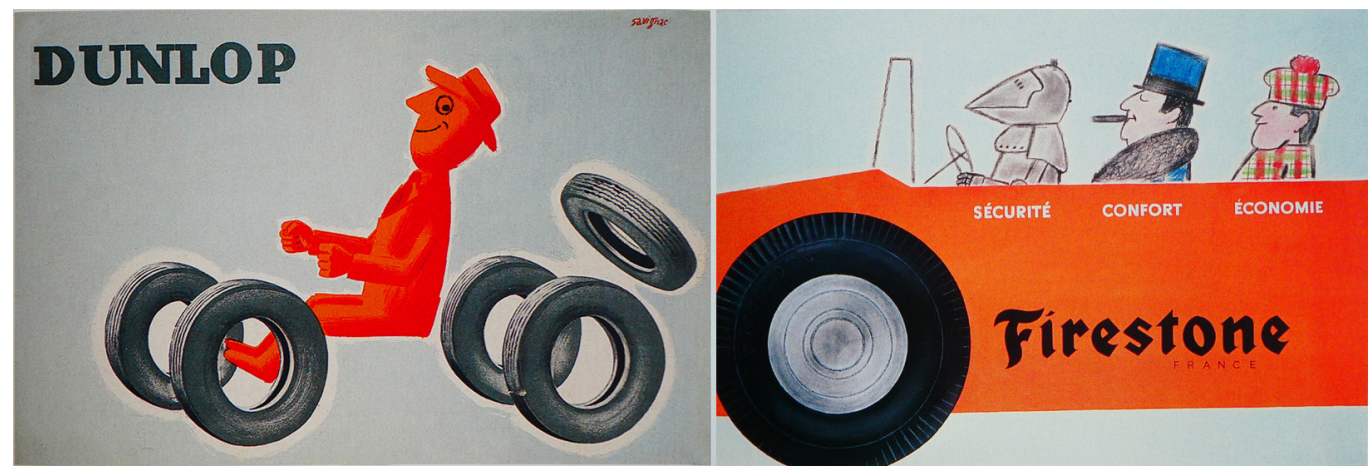

Schueller, the owner of L'Oréal (and Monsavon), who in 1943 hired him at the Consortium Général de Publicité.

In the following years, he produced labels, leaflets, brochures, many sketches, as well as posters for the Consortium: Armagnac Ryst, Armagnac Barnabé, Dalle beer; he also illustrated books, drew cartoons for magazines, created advertising pages and covers.

In 1947, the relationship with the Consortium ended, and the following year Savignac met Bernard Villemot [9]; who, although almost his age, was already an established poster artist. Since the Liberation in 1945, he had already made himself known to the general public, and with some pupils from the Paul Colin [10], school, of whom he had been a pupil in his youth, he opened a studio in rue Danièle-Casanova. Bernard offered Raymond the opportunity to move into his studio, where Savignac felt at home and stayed for several years.

At the beginning of 1949, Villemot, in a crisis of commissions, suggested to his colleague that he prepare an exhibition to promote some of their works, sketches and posters. Savignac contacted a friend of his, the architect Picot, whom he met at the time of the "guerra divertente" and who ran a gallery attached to the École des Beaux-Arts: he obtained the space for a few payments. Raymond drew the poster for the exhibition, using only three colors: black, blue and red.

To make it clear that two authors were exhibiting, he drew two men who vaguely resembled each other, joined by a panel like a sandwich man. On the panel he writes the texts and on the head of one of them he paints a little bird. To characterize them further he draws one with a moustache. Savignac wrote about this detail: "Né Villemot né io abbiamo baffi. Quindi, per assomigliare al mio disegno, mi son lasciato crescere i miei. È proprio da allora che porto i baffi" [Savignac 1975, p. I56].
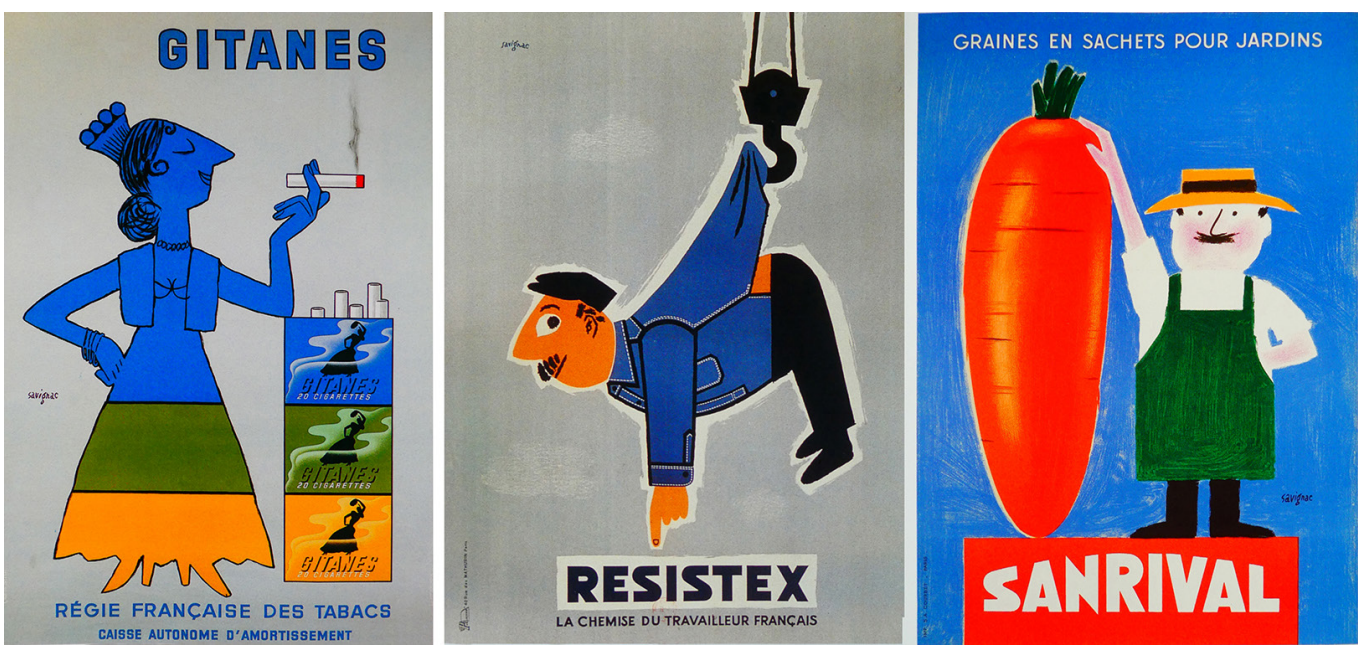
The exhibition opened on the $20^{\text {th }}$ of May 1949 and remained open until the $4^{\text {th }}$ of June. In order to exhibit it in the gallery, Raymond asked Robert Guérin for one of his drawings for the Monsavon advertisement, a sketch that had been lying in a drawer at the Consortium [I I ]. for several months. When Eugène Schueller, the owner of L'Oreal, saw it on display at the exhibition, he was struck by it and decided to print it immediately. It was a huge and immediate success. The city of Paris was invaded by Monsavon's cow.

"Di solito inizio con due idee che si fondono in una sola. Quindi per Monsavon au lait, ho semplicemente pensato a un sapone per Monsavon e una mucca per il latte. Avrei quindi potuto sovrapporre un sapone su una mucca o viceversa: una banalità [... . N Nel mio caso era il collegare questi due immagini così lontane con un elemento che dava vita e logica al mio poster. II latte che schizza dalle mammelle e si trasforma in sapone era il legame che lo rendeva evidente" [Lelieur, Bacholet 200 I, p. 24].

From one day to the next, Savignac became a top poster designer and was inundated with orders. In no time at all, his style became established, acclaimed, imitated, he entered the history of posters, and from then on, with his strong personality, he became a reference point for a whole generation of poster designers. During his career, Savignac worked for more than three hundred and fifty clients [12] producing more than 1000 posters, covers and advertising pages.
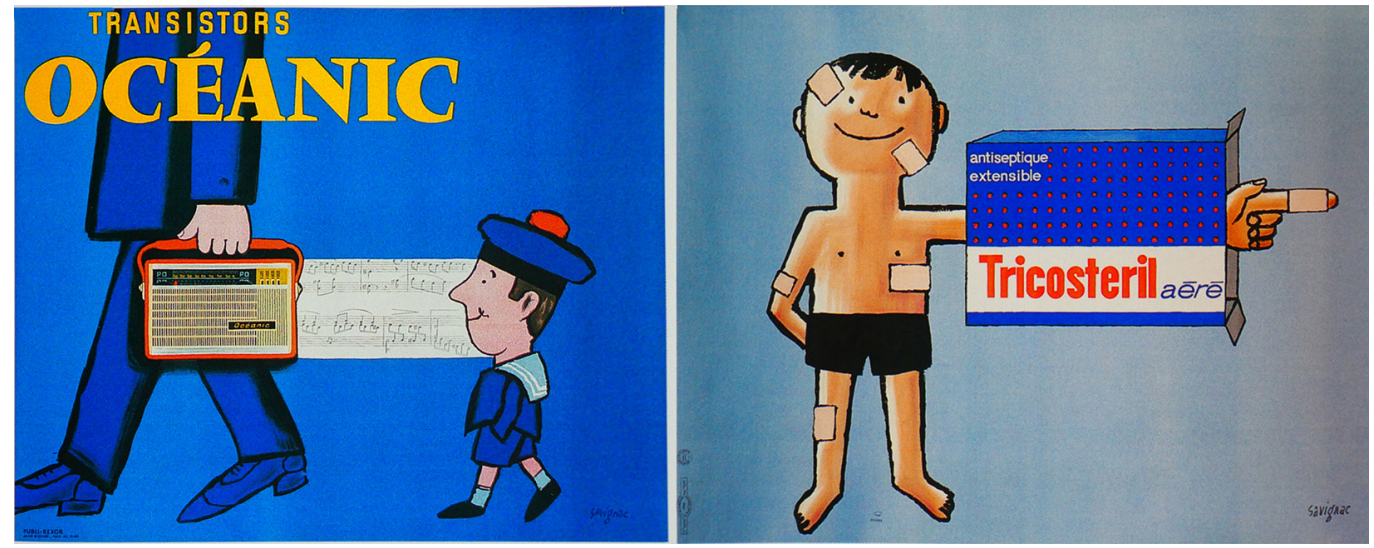

\section{The poster for Savignac}

The poster is ephemeral. Like a newspaper that is thrown away after being read, the poster is torn, covered, replaced, forgotten after fulfilling its function: to inspire a certain gesture or action in those who have looked at it. The first law of the poster is therefore to leap to the eye, the second is that it must be assimilated and understood without effort. An effective advertisement must be noticed immediately, it can even be aggressive, but it must not confuse, repel or provoke scandal; instead, it must be assimilated and acquired almost as soon as it is first seen.

In our society, the poster is used not only to sell, but to make people dream, to make us desire or reject something, it is nothing more than a way of directing our desire. "Come l'opera d'arte, il poster è legato alla forza attrattiva dell'immagine. Ma, a differenza dell'opera d'arte, il poster deve essere visto e letto lo stesso giorno" [Gaëtan-Picon 1978, p. 7]. Savignac works alone,"non crede nell'efficacia di un'idea pubblicitaria partorita da un gruppo, in una sorta di brainstorming all'americana" [Citéra 1998, p. 5]. He is basically an anarchist [13]. He has no pupils, he is not interested in teaching, "prima di tutto perché non ho pazienza, e poi perché non credo affatto nella didattica in questo campo. Se un individuo è intelligente, sensibile, dotato, non ha alcun bisogno degli altri. Se è piuttosto limitato, la cosa non cambia" [Bauret 1982]. The strength of a poster lies in the enterprise of the individual, who is at the same time designer, graphic designer, creator of the slogan and creator of the image. 
His conception of communication is clear:"ll poster non dovrebbe essere solo la trasposizione grafica di un'idea pubblicitaria, ma, prima di tutto, un messaggio di ottimismo; rivolgendosi al grande pubblico, non può preoccuparsi delle reazioni individuali, è quindi condannato a un linguaggio schematico, chiaro e brutale, che non lascia spazio ad aneddoti e sottigliezze" [Lelieur, Bacholet 200 I, p. 16]. Savignac can be considered the inventor of the "gag grafica" or "gag visiva", an operation which consists of advertising and selling through joy, to the pleasure of laughter. He himself states, "quello che ho cercato di inserire nei miei poster è il buon umore" [Weill I987, p. I I]. The development of a "gag grafica" is an exercise in great rigor and high acrobatics: the poster must contain a humor that is simple but not banal and, at times, ironic. If a great actor must make the screen explode, the good advertiser has to "sfondare il muro" [Lescure 20 I4, p. 54]. The "gag visiva", after all, is a means of graphic writing to provoke a shock in passers-by, both in terms of the curiosity it can generate and the mirth it can provoke. Advertising must, however, remain an image, not be too intellectual and be cheerful and energetic: "Dobbiamo tornare a cose semplici, quasi elementari, per ritrovare la salute" [Lelieur, Bacholet 200 I, p. 23]. Savignac never sought to be a communication historian, sociologist or theorist, even though his discourse reveals an organized reflection on society and on the way to convince or condition it through advertising. He was and will always remain only a poster artist.
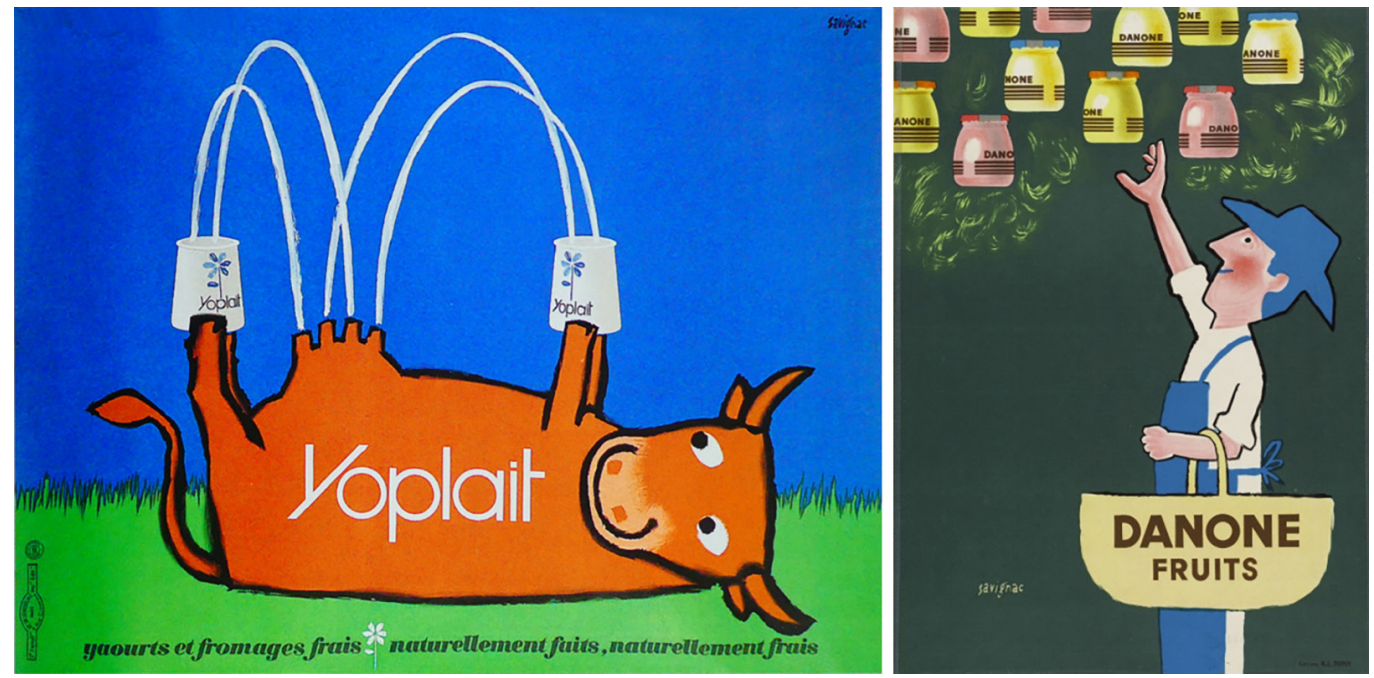

\section{The drawing for Savignac}

For Savignac, the drawing is the "la spina dorsale" of the poster, it has to be dynamic, to bring the idea to life. His posters look almost like sketches, schematic, his figures are surrounded by a black line, almost reminiscent of children's coloring books. In his works the superfluous is absent, erased, it almost seems as if some of his posters are already finished with just the drawing: "Meno mostriamo, più diciamo" [Weill 1987, p. 40].

Savignac understood that, in order to make himself understood, it is advisable to forget the nuances, the realism of the details, in favor of an essential drawing, immediately legible, showing only what is strictly necessary. But Savignac is also a talented colorist, the color being an element added to the sign to make it more vigorous and pleasant. He knows how to set his characters against a background that makes them stand out: blue, red or yellow, basic colors, but skillfully used, trying to harmonize the colors with the product to be advertised. He plays at "coloring" his drawings, like children in their albums [Weill 1987, p. 9]. 
Fig. 10. Savignac: on the left, Frigéco, 1958; on the right, Laden automatique, 1965
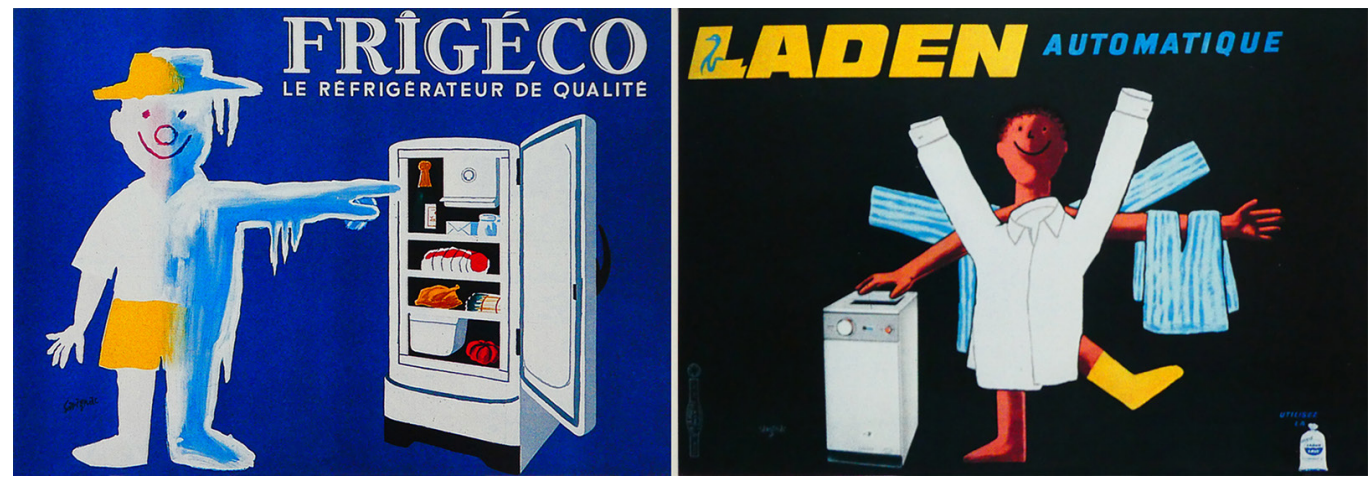

In many of his figures we can see the almost disappearance of relief effects, the characters stand out against the background highlighted by a white or black halo.

His weaknesses are paradoxically his strengths, his naïve style, for example; he defines himself as "grezzo e primitivo" [Weill 1987, p. 8]. Therein lies Savignac's first secret.To this end, his layouts are simple, far removed from the elaborate compositions of his master Cassandre. The schematic graphic layout of Savignac's posters seems almost reminiscent of the concept of the ideogram: "non l'ho inventato io, è antico quanto la Cina stessa. Sia Cassandre che Carlu già l'avevano sperimentato: quando cominciava a svilupparsi nella stampa il sistema del disegno senza didascalia" [Bauret 1982; Weill 1987, p. 9]. Savignac gives the impression that the poster was drawn by a child, the pencil stroke is almost childlike, but with great authenticity. It is a freshness, a spontaneity that everyone loses when they become adults. He has rediscovered it with all the science of an experienced graphic designer and his drawing does not change over time.

Good advertising must entertain the user with the help of striking images, which can sometimes be associated with good slogans. But if the poster with its shape alone achieves the result, the text of the slogan becomes even superfluous. The simple, almost childlike essence of Savignac's graphic language is easy, direct and immediate to understand and if an advertising message can be understood by a child, it will surely reach everyone.

\section{Conclusions}

Savignac introduced original ideas, new schemes and models of communication into the style of modern poster art. He conveyed the commercial message in essential language for immediate readability. The drawing is the basis, the representation must be synthetic, without slogans (which are however inserted into the image), the colors must be bright to attract the observer.
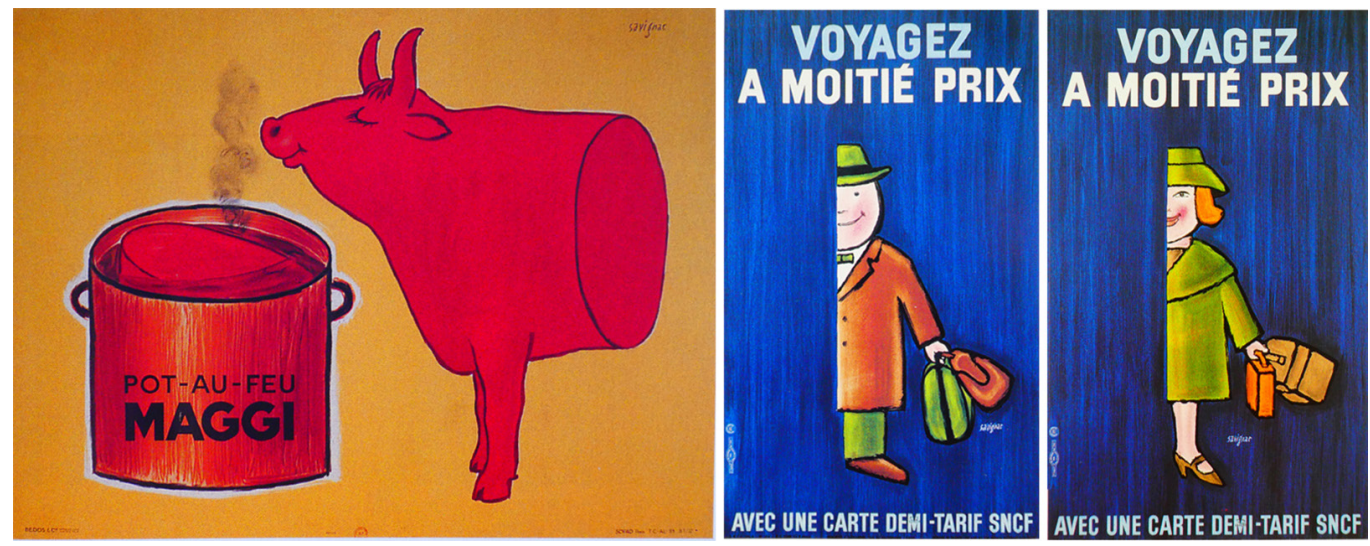

Fig. I I. Savignac: on the left, Brodo Maggi, 1959; in the centre,Viaggi a metà prezzo SNCF, 1964; on prezzo SNCF, 1964 
Savignac communicates optimistic messages with his amusing "gag grafica". His characters always have smiling faces, they convey serenity and effectively address the commercial message. Savignac has always rejected the depersonalization of advertising which uses photography as a communicative device.

Paraphrasing his ideal masters of comic cinema, such as the Charlot and Buster Keaton, Raymond Savignac "ha passato la vita disegnando barzellette" [Olsen 2008, p. I2].

"Noi cerchiamo in primo luogo di far ridere i bambini e senza volerlo facciamo ridere anche $i$ grandi. Che ci possiamo fare se i grandi restano sempre bambini" [Stan Laurel and Oliver Hardy].

Fig. 12. Savignac: on the left, OMO, 1963; in the centre, il Giorno, 1960 on the right, Vespa, 1955
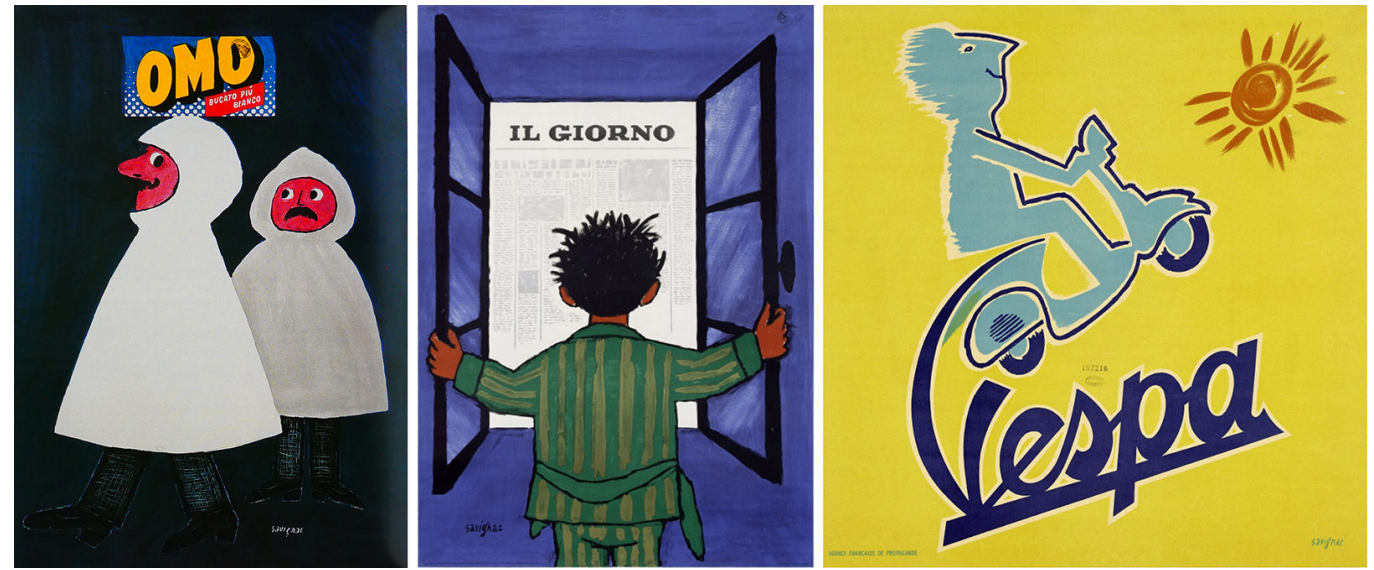

Notes

[I] Savignac says: "Sono un autodidatta, mi sono formato da solo disegnando di sera. Mi divertivo a riprodurre i disegni altrui: era bello e interessante" [Bauret 1982].

[2] In Lortac's studio, Savignac began to understand the work of the poster artists: Cassandre, Loupot, Carlu, Cappiello and Derain, and learned about the art of Derain, Matisse, Picasso [Bauret 1982].

[3] The only cartoon Savignac made was in 1959 for Brodo Maggi [Lelieur, Bacholet 200 I, p. 58].

[4] Adolphe Jean-Marie Mouron, better known as A. M. Cassandre, was born in 1901 in Kharkiv, in the Russian Empire (now Ukraine), into a family of French merchants. He studied painting in Paris, first at the Lucien Simon atelier, then at the Paris Academies de la Grande Chaumière and Julian. His posters, between the 1920s and 1930s, are marked by movement and speed, his clients are: railway companies, sleeping cars, liners, cars and bicycles. For the general public, his name remains associated with a famous advertising campaign of 1932, for a cinchona wine: Dubo, Dubon, Dubonnet in which he creates the little man with the bowler hat who is colored while drinking the liqueur. In 1938, now famous with his agency l'Alliance Graphique, Cassandre moved to the USA for two years. Back in France, in addition to painting and advertising, he designed costumes and theatre sets. He designed the famous 196I logo for the Yves Saint Laurent fashion house. Depressed, partly disillusioned, stressed by work commitments and clients, he committed suicide in 1968.

[5] Alliance Graphique was more of a creative workshop than an advertising agency, founded in 1930 by Cassandre, Charles Loupot and Maurice Moyrand as commercial director. After Loupot's sudden death on the $15^{\text {th }}$ of September 1934 in a car accident (Moyrand was the group's PR man, in charge of client relations and poster sales) and Loupot's gradual withdrawal, Cassandre moved his workshop to his house in Versailles.

[6] "Cassandre è l'unico il cui verdetto accetterò, qualunque esso sia" [Savignac 1975, p. 94].

[7] Cassandre must have sensed the determination of the young Raymond, six years younger, and immediately entrusted him with a number of assignments. His first works in 1933 were the poster for the cheese Roquefort Maria Grimal, Londres for Paul Morand and Cie du Chemin de Fer du Nord [Lelieur, Bacholet 200 I, p. 92].

[8] On the 22 nd of February 1940, Savignac married Marcelle Andréa Mercier, a milliner he had met the year before. "L'unica donna che crede nel mio talento" [Savignac 1975, p. 135].

[9] Bernard Villemot, or more simply Villemot, was born in Trouville-sur-mer in 191।, his father Jean was an illustrator and humorist. As a child he showed a real talent for drawing and after education in the local schools in Paris he undertook art studies at the Academie Julian. After doing his military service in 1931, he attended Jean Colin's studio from 1932 to 1934. Towards the end of 1934, he opened his own atelier with some of the pupils he had met in Colin's studio. From 1935 onwards he began to make his first film posters and exhibition posters. In the 1940s he made posters for the Youth Secretariat and the Vichy 
government. But it was in 1945, with the La Pace ritrovata posters, that his original style emerged:Villemot signed advertising campaigns for the Red Cross and for social themes (aid for children, the fight against alcoholism, cancer, assistance for veterans and the elderly), for public health, for the Armed Forces and for Tourism. Villemot created countless posters, which have remained in the collective memory, for major brands of tobacco, household appliances, food, liquor, cosmetics, cars, engines and much more. In 1953, he began his collaboration with Orangina, which lasted almost twenty years: the orange peel cut into a spiral remained the undisputed icon of the drink for years. His campaign for Perrier began in 1956, marked by the famous slogan "c'est fou...". From 1969 onwards he worked for Bally producing some historic posters: the famous orange lozenges the long white legs, the black backgrounds that become clothes, the sinuous silhouettes, remain some of the most beautiful of his career.The artist died in 1989 in his hometown of Trouville-sur-mer.

[10] Paul Colin was an accomplished poster designer born in Nancy in 1892, he studied at the École des Beaux-Arts, then studied under Eugène Vallin and Victor Prouvé. In 1929 he opened his own studio, where artists such as Villemot, Leupin Henrion, Carrier, Derome and the Lefor-Openo duo were trained. During his career he produced posters for theatre and cinema, posters for musical performances and concerts, including the famous poster for the Revue nègre at the Music-Hall with Joséphine Baker, whose lover he was. Passionate about music and especially jazz, a prolific illustrator, he depicted his characters, artists, dancers and singers, in an admirable formal synthesis that was extremely characterizing. He drew important advertising campaigns for S.N.C.F., Transatlantique, Philips and Columbia. In 1946 he designed the poster for the first Cannes Film Festival. A prolific artist, he produced around I,900 posters, playbills, magazine covers, many theatres sets and costumes. He died in Nogent-sur-Marne in 1985.

[I I] Robert Guérin commissioned Savignac to do a sketch for Monsavon au lait a few months earlier, but the poster, although paid for, was not published and caused some concern. It remained in L'Oréal's drawers. On visiting the exhibition, Eugène Schueller, when he arrived in front of the poster of the Monsavon cow, turned to Savignac and almost moved, exclaimed "Ah Savignac, è meraviglioso il poster chi mi hai fatto" [Savignac 1975, p. I57]

[12] Some of these are international brands: Air France, Air Wick, André, Aspro, Astral, ATD, Bic, Cinzano, Citroën, Collie Mischungs-Treu, Compagnie Wagons Lit France, Crédit Industriel et Commercial, Dalle, Danone, Dop, Dunlop, Dunlopillo, Eau écarlate, French Tobacco Company, Frigeco, Garap, II Giorno, Gendarmerie National, Laine d 'Aoust, Life, Lotterie National, Maggi, Michelin, Monsavon, Mobil, Océanic, Omo, Perrier, Petit Bateau, Pirelli, Point P., Renault, Résistex, Reynolds, Salveplast, Savings Bank, Seb, Solutricine, SNCF, Tréca, Triplex, Tricostéril, Ultracolor, Vérigoud, Vichy Célestins, Yoplait. Savignac also works for public bodies and associations, for opinion movements, such as - for example - the famous poster created for the awareness campaign "No alla superstrada sulla riva sinistra" of 1971.

[13] This is how he defines himself A. Weill [Weill 1987, p. 13]. And indeed, in his profession Savignac has a decidedly anarchic character, apart from the period when he used Villemot's studio, from 1948 to 1953, he always worked alone. Apparently he was neither interested in politics [Savignac 1975, p. 5 I], nor in political satire "Il disegno politico non è il mio pezzo forte" [Savignac 1975, p. 165]. Twelve years later, he is tempted by some right-wing satirical editions, but not for long [Lelieur, Bacholet 200 I, p. 69].

\section{References}

Bauret G. (1982). Intervista a Savignac. In Zoom, n. 22, settembre, p. 43 ss.

Choko M. H. (2005). Raymond Savignac. Paris: Pyramyd.

Citéra F. (1998). Savignac en vrac. Cabourg: Ed. Cahiers du Temps.

Decaen Le Boulanger H. (2008). La revolution Savignac. Cabourg: Ed. Cahiers du Temps.

Gaëtan-Picon G. (1978). In Musée de l'affiche. Paris: s.d. ma.

Lelieur A. C., Bacholet R. (200 I). Savignac affichiste. Paris: Ed. Bibliothèque Forney.

Lescure P., Buchard S. (20|4). Marques et Pubs cultes. Paris: Éd. De La Martinière.

Olsen R. (2008). Savignac s'affiche. Paris: Le Cherche midi.

Savignac R. (1975). Savignac affichiste. Paris: Ed. Robert Lafont.

Weil A. (1987). Savignac. L'affiche de A à Z. Montpellier: Seuil - Édition Hoëbeke.

Author

Marcello Scalzo, Università degli Studi di Firenze, marcello.scalzo@unifi.it

To cite this chapter. Scalzo Marcello (2021). Riflessioni sul linguaggio grafico nei poster di Savignac/Reflections on the graphic language of Savignac's poster. In Arena A., Arena M., Mediati D., Raffa P. (a cura di). Connettere. Un disegno per annodare e tessere. Linguaggi Distanze Tecnologie. Atti del $42^{\circ}$ Convegno Internazionale dei Docenti delle Discipline della Rappresentazione/Connecting. Drawing for weaving relationship. Languages Distances Technologies. Proceedings of the $42^{\text {th }}$ International Conference of Representation Disciplines Teachers. Milano: FrancoAngeli, pp. I I 23 - I I 42. 